\title{
¿Techos o Suelos?: Diferenciales salariales por género y educación en España*
}

\author{
Sara de la Rica \\ Universidad del País Vasco \& IZA
}

Juan J. Dolado

Universidad Carlos III \& CEPR \& IZA

Vanesa Llorens

LECG Consulting Spain S.L.

\section{Resumen}

En este trabajo se analiza la forma como varía el diferencial entre los salarios de hombres y mujeres a lo largo de la distribución salarial utilizando datos del PHOGUE. Se utilizan regresiones cuantílicas y métodos de panel para estimar ecuaciones salariales en los percentiles relevantes para un año representativo de la muestra (1999) y a lo largo del tiempo (1994-2001). A diferencia de otros países donde el diferencial tiene un perfil creciente a lo largo de la distribución, la evolución no monotónica de dicho gap en España se explica por un interesante efecto de composición cuando se divide la muestra de trabajadores por nivel educativo. Para los trabajadores con mayor nivel educativo, en línea con la conocida hipótesis de los techos de cristal, el diferencial aumenta conforme nos movemos hacia los percentiles superiores. Sin embargo, nuestro principal resultado muestra que el diferencial para los trabajadores con menor nivel educativo tiene una apreciable pendiente decreciente, es decir, es mucho mas elevado en la parte baja que en la parte alta de la distribución salarial. Dicha pendiente resulta incluso más negativa cuando se corrige por sesgos de selección muestral y se mantiene relativamente inalterada cuando se controla por las diferentes características productivas de los trabajadores. Denominamos a este nuevo fenómeno patrón de suelos, argumentando que puede explicarse por la discriminación estadística que ejercen los empresarios en aquellos países con baja participación laboral de las mujeres con menor nivel educativo.

Palabras clave: gaps entre géneros, cielos de cristal, suelos de cristal, regresiones cuantílicas. Clasificación JEL: J16, T17

\begin{abstract}
This paper analyses the way in which the gender gap varies throughout the wage distribution in Spain using data from the ECHP. Quantile regression and panel data techniques are used to estimate wage equations at relevant percentiles in a representative year of the sample (1999), and during the period (19942001). In contrast with other countries, where the wage differential exhibits an increasing pattern throughout the distribution, the flatter evolution of gender wage gap in Spain is explained by a composition effect when the sample of workers is divided by education levels. For high-educated workers, in line with the conventional glass ceiling hypothesis, the gap increases as we move up to the highest percentiles.
\end{abstract}

* Este artículo es una traducción de la versión actualizada del trabajo aparecido como Discusión Paper no. 1483 del IZA. Estamos agradecidos a una Editora y tres evaluadores anónimos del Journal of Population Economics por sus comentarios. 
However, our main result shows that the wage gap for less-educated workers is decreasing, that is the wage gap is much higher at the lower than at the upper part of the distribution. This pattern is even more acute when we correct for sample selection bias and remains relatively unaltered when we control for differences workers' characteristics. We label this phenomenon as a floor pattern and argue that it can be explained by statistical discrimination exerted by employers in countries where less-educated women have low participation rates.

Keywords: gender gaps, glass ceiling, glass floors, quantile regressions

JEL Classification: J16, J71

\section{Introducción}

Un fenómeno ampliamente documentado es que los hombres perciben salarios más elevados que las mujeres, incluso controlando tanto por las diferencias en características productivas observables como por la evolución de la distribución salarial en su conjunto (véase, p. ej. Blau y Kahn, 2000). Existe una amplia literatura sobre este tema basada en la comparación de medias salariales (en logs.), donde el tamaño del diferencial salarial (en adelante, gap entre géneros) se interpreta típicamente como una estimación de la discriminación existente en la media de la distribución observada de salarios. Sin embargo, el análisis del gap entre géneros en otros puntos de la distribución ha despertado menos interés ${ }^{1}$. No obstante, en los últimos años esta situación está cambiando y un buen ejemplo es el interés creciente por examinar si la discriminación salarial es mas alta entre los trabajadores con mayores salarios que entre aquellos con menores salarios, en línea con la denominada hipótesis de los techos de cristal.

En este trabajo, inspirándonos en el enfoque de Albrecht et al. (2003) para documentar la presencia de techos de cristal en Suecia, derivamos en primer lugar medidas por cuantiles del gap entre géneros en España a finales de los noventa. Posteriormente, contrastamos nuestra explicación de la evolución de los mismos utilizando procedimientos de datos de panel. Éste resulta ser un tema muy relevante puesto que España, al igual que otros países del sur del Mediterráneo (p.ej., Grecia e Italia, pero no Portugal), todavía tiene una participación laboral femenina bastante inferior a la de

1 Ejemplos bien conocidos de este tipo de análisis son Chamberlain (1994) y Buchinsky (1994, 1998a,b) que usan QR para analizar la structural salarial en EE.UU. Una aplicación de este método para España se encuentra en Abadie (1997) y aplicaciones más generales en Fitzenberger et al. (2001). Más recientemente, dichas técnicas de estimación han sido utilizadas para examinar la discriminación salarial por género en los antiguos países comunistas (Newell and Reilly, 2001) y en Suecia (Albrecht et al., 2003). Con respecto a este ultimo tema en España, existen dos trabajos relacionados con el nuestro. Por una parte, García et al. (2001), usando la Encuesta de Conciencia, Biografía y Estructura de Clase de 1991, controlan tanto por la endogeneidad de la educación como por los sesgos de selección de las mujeres trabajadoras, concluyendo que el componente de discriminación, en términos absolutos y relativos, es superior en la parte alta de la distribución salarial. Por otra, Gardeazabal y Ugidos (2002), utilizando la Encuesta de Salarios de 1995, también encuentran que el gap salarial entre géneros también aumenta a lo largo de la distribución pero, por el contrario, estiman que el componente de discriminación, en términos relativos, es mayor en la parte baja de la distribución. 
los países Nórdicos, por lo que los logros de las mujeres en el mercado laboral en aquellos países seguramente difieren sustancialmente de lo que acontece en estos últimos $^{2}$. En efecto, la evidencia que mostramos en este trabajo sustenta esta conjetura: el gap de géneros global a lo largo de la distribución tiene un perfil mucho mas aplanado en España que en Suecia. En este sentido, siendo ésta nuestra contribución más importante, desvelamos un interesante efecto de composición detrás de este perfil cuando la muestra de trabajadores se divide por nivel educativo ${ }^{3}$. Conforme nos movemos hacia la parte más alta de la distribución salarial, el gap entre géneros para los trabajadores con educación superior (universitaria; en adelante grupo $\mathrm{H}$ ) aumenta. Por el contrario, en el caso de los trabajadores con nivel educativo inferior (primaria/secundaria; en adelante grupo L), el gap disminuye. Mientras que el gap creciente para el grupo H concuerda con los resultados previos de Albrecht et al (2003) para Suecia, la pendiente negativa del gap para el grupo L es un hecho novedoso en esta literatura con implicaciones interesantes de política económica. Con el fin de acentuar sus diferencias con los convencionales techos de cristal, en el resto del artículo se utilizará la nomenclatura de perfil de suelos para designar a este nuevo fenómeno ${ }^{4}$.

A continuación, se ofrece una ilustración preliminar de estos hechos utilizando la sexta ola (1999) del Panel de Hogares de la Unión Europea (PHOGUE), utilizando una muestra de trabajadores a tiempo completo ${ }^{5}$. La Figura 1a muestra el gap entre géneros (en términos de diferencias logarítmicas de los salarios brutos por hora entre hombres y mujeres) en España a lo largo de la distribución salarial, conjuntamente con el gap medio (línea discontinua) ${ }^{6}$. Se observa que existe una tendencia decreciente que desaparece a partir del decil 60 para, posteriormente, convertirse en positiva en los cuantiles superiores. Como es predecible, el gap medio difiere sustancialmen-

2 La tasa de actividad femenina en España (\% de la población con edad entre 15-64) en 2001 era del $50.7 \%$ mientras que alcanzaba $75.7 \%$ en Suecia y $60.2 \%$ en la UE Por niveles educativos, las tasas correspondientes en España eran del $80.4 \%$ y $48.0 \%$ par alas mujeres con educación universitaria y menos de dicho nivel (84.6\% y 68.3\% en Suecia), respectivamente (véase OECD, 2002). De hecho, el grupo de mujeres trabajadoras en España está formado por cohortes muy heterogéneas. Desde los ochenta, la participación laboral femenina ha aumentado mucho (desde el $33.3 \%$ en 1980 hasta el 50.7\% en 2001) principalmente debido a un fuerte incremento en el acceso a la educación universitaria y a una reducción en la tasa de fertilidad. (véase, p.ej., Arellano and Bover, 1995).

3 Los niveles educativos se tratan como categorías predeterminadas a lo largo de este trabajo ya que nuestro objetivo no es estimar sus rendimientos.

${ }_{4}$ Un nombre más apropiado sería el de «techos de cristal en la planta baja» puesto que se refiere al gap salarial en los cuantiles bajos de la distribución. Puesto que el término «suelos de cristal» podría interpretarse erróneamente como un impedimento a que los salaries femeninos cayeran demasiado bajo, el término «patrón de suelos» se utilizará en lo que sigue por su brevedad. Nótese que este concepto no debe confundirse con el de «suelos pegajosos», concepto relacionado con los salarios inferiores a los de los hombres que reciben las mujeres en la parte alta de la distribución debido a la escasez relativa de ofertas salariales de otras empresas para las trabajadoras (véase Booth et al., 2003). En un marco no-competitivo donde las rentas se reparten entre trabajadores y empresarios, un salario alternativo superior para los hombres podría situar sus salarios por encima de los de las mujeres igualmente productivas.

5 Se observan patrones similares en las otras olas disponibles del PHOGUE.

6 Los percentiles comparados se refieren a las distribuciones de hombres y mujeres separadamente. Si se considera la posición de las mujeres en la distribución de los hombres, se encuentra que un $31 \%(5.4 \%)$ de las mismas está en el decil inferior (superior). 
te del gap a lo largo de la distribución. Nótese que dicha evolución no monotónica difiere sustancialmente de la existente en Suecia (Figura 1b) donde el gap se incrementa en 35 puntos porcentuales desde la parte baja a la parte alta de la distribución, en línea con el fenómeno de techos de cristal documentado por Albrecht et al. (2003).

FIGURA 1a
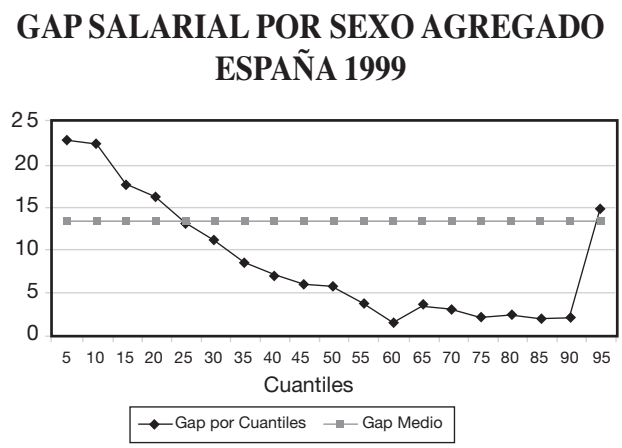

FIGURA 1c

GAP SALARIAL POR EDUCACIÓN GRUPO H ESPAÑA 1999

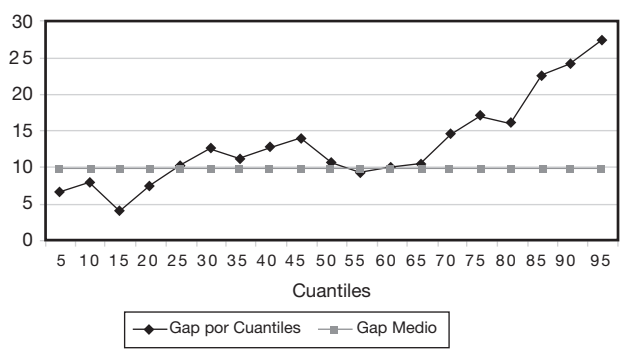

FIGURA 1b
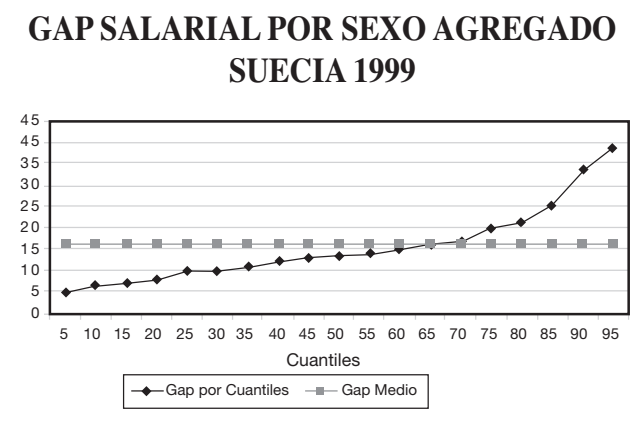

FIGURA 1d

GAP SALARIAL POR EDUCACIÓN GRUPO L ESPAÑA 1999

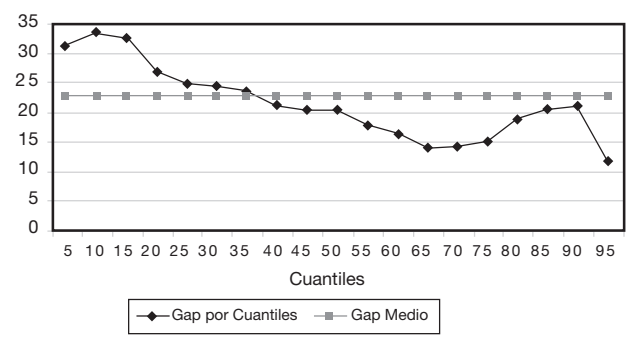

Las Figuras 1c y 1d, a su vez, muestran los correspondientes gaps por cuantiles para los grupos L y $\mathrm{H}$ de trabajadores españoles. El gráfico para el grupo $\mathrm{H}$ es creciente, de manera que recuerda a los techos de cristal. Por el contrario, el gap para el grupo L es decreciente. Por tanto, la agregación de ambos grupos conduce al perfil más aplanado y no monotónico mostrado en la Figura 1a. Parece pues haber un interesante efecto de composición educativa que merece analizarse con mas detalle. Resulta interesante corroborar que los países del norte y centro de Europa, como Dinamarca o Reino Unido (Figuras 2a y 2b), exhiben gaps monotónicamente crecientes para ambos grupos educativos, como ocurría en Suecia, mientras que en los países del sur de Europa con reducida participación laboral femenina, como Grecia e Italia (Figuras 2c y 2d), los perfiles son más irregulares. No obstante, los gaps para 
FIGURA 2a

\section{GAP SALARIAL POR EDUCACIÓN DINAMARCA 1999}

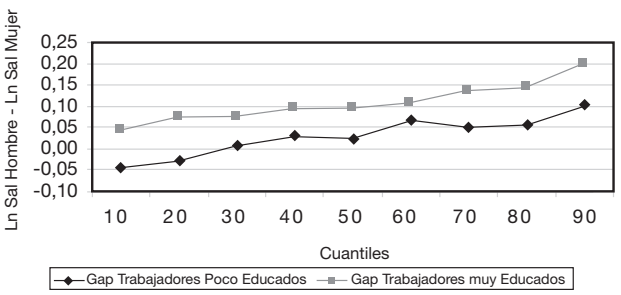

FIGURA 2c

\section{GAP SALARIAL POR EDUCACIÓN GRECIA 1999}

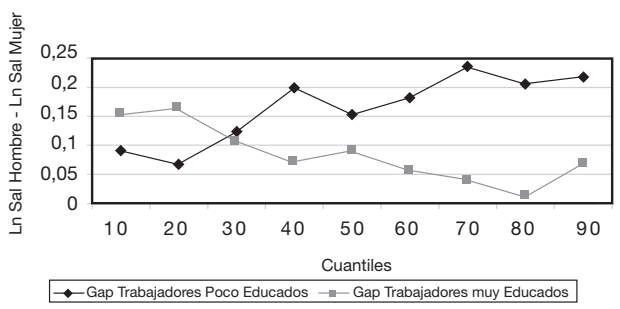

FIGURA 2b

\section{GAP SALARIAL POR EDUCACIÓN REINO UNIDO 1999}

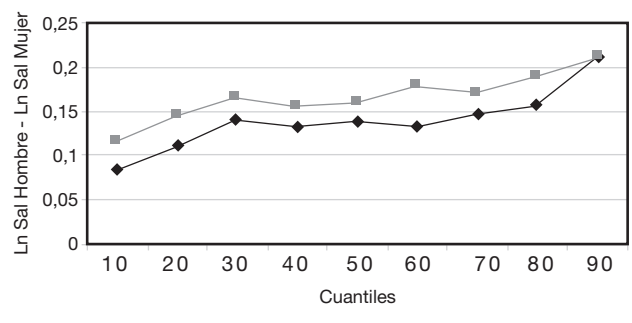

- Gap Trabajadores Poco Educados - $\rightarrow$ - Gap Trabajadores muy Educados

\section{GAP SALARIAL POR EDUCACIÓN ITALIA 1999}

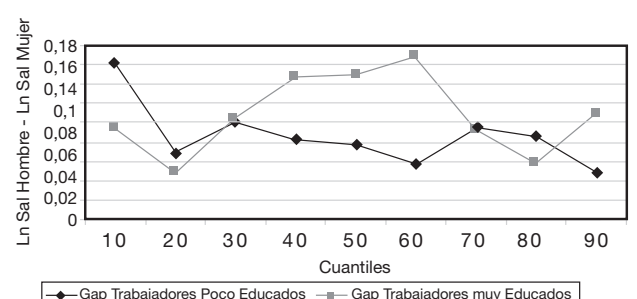

el grupo L en ambos países tienen un perfil de suelos similar al documentado para España ${ }^{7}$.

Existen varias explicaciones para reconciliar estos patrones divergentes del gap entre géneros por nivel educativo: ${ }^{8}$

1. En el caso de las mujeres del grupo L, las tasas de actividad todavía son mucho mas bajas en los países del sur del Mediterráneos que en los países del

7 Los gaps reportados para Dinamarca, Reino Unido, Grecia e Italia corresponden a la ola de 1999 del PHOGUE. El gap para Suecia reproduce la Figura 1 de Albrecht et al. (2003) correspondiente a 1998 con datos procedentes de Statistics Sweden (SSW). Las tasas de actividad en estos países se encuentran en el Cuadro A1 del Apéndice.

8 Otra posible explicación, no mencionada anteriormente, podría surgir de la existencia de alguna forma de heterogeneidad inobservada que afectaran a las mujeres de tipo L en relación a los hombres en este grupo (véase sección 5). Además, la OECD (2002) llama la atención sobre la posibilidad de que hayan errores de medición en la muestra procedentes del hecho de que los individuos entrevistados proporcionan la información sobre sus salarios directamente, en vez de recabarse de las empresas, como es el caso con datos cruzados procedentes de empresas y trabajadores.Si aquellos con salarios mas altos, típicamente los hombres, tienen una mayor propensión a declarar salarios mas bajo, el gap en los cuantiles superiores estaría infraestimado. Aunque este argumento pudiera implicar sesgos a la baja en la parte alta de la distribución para ambos grupos de trabajadores, no puede explicar el patrón hallado en la parte baja de la distribución del grupo L. 
centro y norte de Europa, a pesar del proceso de convergencia en participación laboral con los segundos que ha tenido lugar en el primer grupo de países durante las dos últimas décadas (véase Cuadro A1 in el Apéndice). La baja tasa de participación puede dar lugar a un proceso de selección no aleatoria de las mujeres que trabajan afectando seriamente al gap entre géneros resultante. Por tanto, podría argumentarse que el patrón de suelos es simplemente la consecuencia de un fenómeno puro de selección muestral. En principio, se puede pensar que hay dos formas alternativas en que operan los sesgos de selección ${ }^{9}$. Por una parte, si todas las mujeres de tipo L participan en la parte baja de la distribución salarial - típicamente, como comentaremos después, cuando son jóvenes y su capital humano acumulado es reducido - pero sólo aquellas con mejores características productivas permanecen en el mercado laboral situándose en los cuantiles mas altos (probablemente en etapas posteriores de sus carreras profesionales), entonces el patrón de suelos observado podría deberse a este tipo de selección. En efecto, las trabajadoras con mayor experiencia en los cuantiles medios y altos constituirían un grupo con selección positiva, explicando de esta forma los gaps reducidos en dichas partes de la distribución. Por otra, podría pensarse que la selección es negativa conforme aumentan la experiencia y antigüedad de las mujeres. Este sería el caso si la mayoría de las mujeres trabajara cuando son jóvenes, pero sólo aquellas con mayores necesidades económicas permanecieran en el mercado laboral. Este proceso de selección no podría explicar la pendiente decreciente del gap. De hecho, la corrección por este tipo de selección muestral exacerbaría la pendiente negativa que caracteriza al patrón de suelos. Tal como se muestra en la sección 3, nuestra evidencia apoya al último tipo de selección. Por tanto, argüimos que la selección no aleatoria de los individuos en la muestra no puede explicar por si sola el fenómeno de los suelos.

Una explicación plausible de dicho fenómeno debería, por consiguiente, sustentarse en argumentos distintos al de selección muestral en las partes media y alta de la distribución salarial. La que proponemos aquí se basa en la hipótesis de discriminación estadística cuando se requiere algún tipo de formación específica para desempeñar un puesto de trabajo. La idea es que, en la medida en que las mujeres con menor nivel educativo experimentan interrupciones frecuentes en su vida profesional - debido a discriminación social en las tareas del hogar, escasez de ayudas a las familias, o creencias religiosaslos empresarios pueden usar procedimientos de discriminación estadística a la hora de establecer el salario de una mujer cuando se la contrata. Actuarían así para evitar pérdidas en caso de abandonos inesperados una vez que hayan financiado una parte de la formación de este tipo de mujeres. En otras palabras,

9 En cualquier caso, evidencia de que el sesgo de selección afecta al gap medio en los países del sur del Mediterráneo (véase Olivetti y Petrongolo, 2005). La cuestión importante, sin embargo, es cómo afecta a la pendiente del gap a lo largo de la distribución salarial. 
si la formación específica se requiere en las etapas iniciales de un empleo, cuya financiación es compartida por empresario y trabajador, aquel puede decidir financiar una proporción mas baja de la formación específica de la mujer que del hombre. Este fenómeno resultará en salarios femeninos más reducidos que los masculinos en la parte baja de la distribución, la cual, típicamente, captura los salarios pagados en los primeros trabajos de la vida profesional. Sin embargo, conforme aumenta la antigüedad en el puesto de trabajo, la incertidumbre asociada a la inestabilidad de una mujer en el puesto de trabajo desaparece, de manera que sus salarios, a igualdad de características productivas, tenderían a equipararse a los de los hombres.

2. Las mujeres del tipo $H$, por contra, presentan unas tasas de actividad mucho más altas, solo ligeramente por debajo de las masculinas de tipo $\mathrm{H}$, incluso en los países del sur del Mediterráneo (véase Cuadro A1), y presentan una baja propensión al abandono dada la elevada inversión en capital humano que han llevado a cabo. Por tanto, ni la selección muestral ni la discriminación estadística parecen explicaciones apropiadas para justificar el gap salarial en este grupo de trabajadores. En consonancia con la percepción de los empresarios de que el compromiso de estas mujeres con el puesto de trabajo es tan alto como el de los hombres, los salarios femeninos serán similares a los masculinos al comienzo de las respectivas carreras profesionales que, típicamente, corresponde a la parte baja de la distribución salarial. Sin embargo, conforme nos movemos hacia los cuantiles superiores, el gap en favor de los hombres aumenta si se produce el tradicional fenómeno de los techos de cristal para el que se han propuesto varias explicaciones en la literatura ${ }^{10}$.

Puesto que los techos de cristal constituyen un fenómeno bien documentado, nos concentraremos en el resto de este trabajo en el nuevo fenómeno: el patrón de suelos para aquellos países con baja participación laboral de las mujeres en el grupo L. Se utilizarán datos españoles para evaluar nuestra interpretación económica de este hecho ${ }^{11}$. Alguna evidencia preliminar en favor de nuestra interpretación preferida puede obtenerse de una reciente encuesta llevada a cabo por el observatorio del Ministe-

10 Por ejemplo, Polachek (1981) predice que las mujeres escogen ocupaciones en las que la penalización por bajas laborales es reducida. La existencia de segregación ocupacional por género apoyaría este argumento (véase Dolado et al., 2004). Otra explicación se basa en el hecho de que las mujeres tengan una menor probabilidad de promoción a puestos de mayor responsabilidad incluso en el caso de que tengan la misma distribución de habilidades que los hombres. El modelo de Lazear y Rosen (1990) asume una mayor productividad a las mujeres en las tareas del hogar, supuesto que reduce la probabilidad de invertir en su formación por parte de los empresarios. Por tanto, solo las mujeres más productivas serán promocionadas. Finalmente, como se mencionó previamente, la explicación del modelo de los «suelos pegajosos» de Booth et al. (2003) descansa en el supuesto de que los hombres reciben un mayor número de ofertas alternativas.

${ }_{11}$ Los detalles de la muestra del grupo $\mathrm{H}$ y el análisis empírico del fenómeno de los «techos de cristal» utilizando el procedimiento de $\mathrm{QR}$ pueden encontrase en la versión previa de este trabajo (véase de la Rica et al. 2005). 
rio de Trabajo y Asuntos Sociales (MTAS) sobre el comportamiento de la mujer trabajadora en España (Instituto de la Mujer, 2005). En concreto, el informe ofrece información sobre la relación existente entre abandonos voluntarios y nivel educativo por género. En base a una muestra estratificada de 4.000 individuos de edad entre 16 y 65 años de la Encuesta de Población Activa (EPA) en 2003, se encuentra que la proporción de abandonos (de al menos un año de duración) entre las mujeres tipo L es del $31.7 \%$ mientras que entre los hombres tipo L alcanza un 14.4\%. Por contra, las tasas de abandono entre mujeres y hombres del tipo $\mathrm{H}$ son del $13.3 \%$ y $8 \%$ respectivamente. Por tanto, la baja tasa de actividad de las mujeres tipo L, relativamente a los hombres tipo L, parece estar positivamente correlacionada con su propensión al abandono laboral. Esta correlación confirma aparentemente las creencias de los empresarios de que las mujeres abandonan con más frecuencia que los hombres, reforzando de esta forma la idea de la discriminación estadística como un fenómeno de equilibrio.

Puesto que el gap representado en la Figura 1d puede deberse a una productividad inferior de las mujeres o a un rendimiento de mercado inferior de unas características productivas dadas, normalmente asociadas a un componente de discriminación o a componentes inobservables, resulta importante separar ambos componentes del diferencial salarial. En este sentido, seguimos dos enfoques econométricos alternativos: uno utiliza datos de sección cruzada (cross-section), para facilitar la comparación con los resultados de Albrecht et al. (2003), mientras que el otro usa datos de panel para controlar por heterogeneidad inobservable. Con respecto al primer enfoque, utilizamos regresiones cuantílicas $(\mathrm{QR})$ - controlando por selección muestral - para obtener los rendimientos de mercado de las características de hombres y mujeres tipo L en un año representativo (1999). Dada la naturaleza de sección cruzada de los datos, la interpretación de los resultados en términos de la explicación propuesta requiere el supuesto subyacente - en favor del cual se proporciona evidencia en la sección 3 - de que edad/experiencia y antigüedad aumenten conforme nos movemos de izquierda a derecha en la distribución salarial, de manera que los cuantiles más bajos reflejen salarios en las etapas iniciales de una carrera profesional mientras que los más altos correspondan a salarios en etapas posteriores. Se utiliza la versión de la conocida descomposición de Oaxaca-Blinder del gap salarial propuesta por Machado y Mata (2004) para el contexto de QR, con el fin de separar los dos componentes comentados previamente. Nuestro principal resultado es que las diferencias en características explican la mitad del gap (original) entre géneros en los cuantiles superiores y solo un $10 \%$ en los inferiores. Además, el componente residual del gap presenta un perfil decreciente similar al del gap original mostrado en la Figura 1d. Especialmente relevante es el resultado de que los rendimientos de mercado a la antigüedad en el puesto de trabajo son más altos para las mujeres que para los hombres en la parte baja de la distribución, y que las diferencias en antigüedad expliquen buena parte del perfil de suelos en el gap contrafactual - construido de manera que hombres y mujeres tengan las características de los primeros. Este resultado resulta ser consistente con la hipótesis de dis- 
criminación estadística descrita anteriormente de acuerdo con la cual, conforme se amplía la antigüedad de las mujeres del grupo L, el gap salarial con los hombres de dicho grupo tiende a desaparecer.

El segundo enfoque econométrico - análisis de datos de panel - sirve para comprobar si nuestros resultados con sección cruzada permanecen cualitativamente similares cuando somos capaces de seguir a los individuos en su puesto de trabajo a lo largo del tiempo. En este caso, el supuesto que relaciona los cuantiles de la distribución salarial con las diferentes etapas en la vida profesional del trabajador no es necesario. Las ocho olas disponibles (1994-2001) del PHOGUE se usan para estimar ecuaciones de salarios (en logs.) mediante efectos fijos con el fin de contrastar si los rendimientos de mercado de la antigüedad son más elevados para las mujeres que para los hombres en el grupo L. Como se discutió antes, si los empresarios penalizan inicialmente a las mujeres por su mayor propensión al abandono en grupos con baja tasa de actividad, una mayor antigüedad de una trabajadora recibiría un rendimiento más alto que la de un hombre en dicho grupo, mientras que dichas diferencias de rendimientos no deberían existir en el grupo $\mathrm{H}$. Este es precisamente el resultado que encontramos al utilizar el enfoque de datos de panel.

El resto del artículo se estructura de la forma siguiente. En la Sección 2, motivamos el análisis mediante un sencillo modelo teórico que es consistente con nuestra explicación del patrón de suelos en el grupo L basada en la hipótesis de discriminación estadística. La Sección 3 se dedica a describir brevemente la metodología econométrica de QR, la base de datos, los efectos de la corrección por selección y los resultados de estimar las ecuaciones salariales por género. En la Sección 4 se desarrolla la descomposición del gap en forma de QR. La Sección 5 explota la dimensión de panel del PHOGUE para corroborar la hipótesis de discriminación estadística. Finalmente, se concluye en la Sección 5. Un Apéndice ofrece una descripción detallada de los datos.

\section{Un modelo de discriminación estadística para los suelos}

Con el fin de justificar la presencia de suelos en la evolución a lo largo de los cuantiles del gap entre géneros para los trabajadores en el grupo L, usamos un sencillo modelo motivado por el trabajo de Acemoglu y Pischke (1998) sobre los efectos de la financiación de la formación específica en mercados laborales con fricciones de búsqueda, adaptándolo a nuestro contexto donde no existen dichas fricciones pero sí existen los elementos de monopsonio que caracterizan el mercado laboral de las mujeres con escaso nivel educativo (véase capítulo 7 en Manning, 2003).

Supongamos que los trabajadores están dotados con un nivel de habilidad $\delta$ cuya función de distribución, $G(\delta)$, es idéntica para hombres y mujeres. Los trabajadores del grupo $L$ requieren una formación específica para desempeñar un puesto de trabajo, de forma que se consideran dos períodos. Dicha formación se ofrece igualmente a ambos géneros. 
En el período inicial, los individuos reciben la formación mientras trabajan (digamos que reciben cursos de formación por las mañanas y trabajan por las tardes). Debido a esta combinación de actividades, las empresas deben pre-anunciar el salario de este período antes de que la formación tenga lugar. Además, las empresas soportan un coste de inversión en formación que conlleva una menor productividad en este período que es igual a $\gamma_{1} \delta$, con $0<\gamma_{1}<1$. En este punto, las empresas desconocen la productividad del trabajador que les es revelada al comienzo del período 2 , una vez que los trabajadores han sido formados. La formación recibida conlleva una mayor productividad $\gamma_{2} \delta$ en el período 2, de forma que se verifica la desigualdad: $\gamma_{1}<1<\gamma_{2}$. Los trabajadores reciben un shock de desutilidad, $\omega$, que les obligue a abandonar el trabajo (digamos que por obligaciones familiares), como en Acemoglu y Pischke (1998). El shock $\omega$ es una variable aleatoria i.i.d. con función de distribución $\mathrm{F}(\omega)$. Dicho shock ocurre dos veces: (i) primero, hay un shock $\omega_{1}$ en el período 1 después de que el salario en dicho período, $\mathrm{W}_{1}$, haya sido anunciado por parte de la empresa; y (ii) posteriormente, hay otro shock $\omega_{2}$ en el período 2 (independiente de $\omega_{1}$ ) después de que la empresa anuncie de nuevo el salario en dicho período, $\mathrm{W}_{2}$. Por tanto, los individuos trabajarán y recibirán formación en el período $1 \mathrm{si}_{1}-\omega_{1} \geq 0$, y trabajarán en el período $2 \mathrm{si} \mathrm{W}_{2}-\omega_{2} \geq 0$. Con el fin de enfatizar el argumento monopsonístico, no se permiten renegociaciones salariales tras los shocks ni ofertas salariales de otras empresas para los que abandonan el puesto de trabajo.

La diferencia clave entre hombres y mujeres es que la función de distribución de los shocks entre los hombres, $\mathrm{F}_{\mathrm{m}}(\omega)$, está estocásticamente dominada por la de las mujeres $F_{f}(\omega)$, esto es: $F_{m}(\omega)>F_{f}(\omega)$ para $\omega>0$. Este supuesto captura el hecho de que las mujeres tienen una mayor propensión a recibir los shocks que los hombres, bien debido a que tienen mayores oportunidades externas en el trabajo en el hogar o por discriminación social. Para simplificar los cálculos, y sin pérdida de generalidad en términos de los resultados cualitativos, supondremos que $\mathrm{dG}($.$) y \mathrm{dF}($.$) son distribu-$ ciones uniformes, de manera que las funciones de densidad verifican: $\mathrm{g}(\delta)=\mathrm{U}[0, \tau]$, $\mathrm{f}_{\mathrm{m}}(\omega)=\mathrm{U}\left[0, \varepsilon_{\mathrm{m}}\right]$ y $\mathrm{F}_{\mathrm{f}}(\omega)=\mathrm{U}\left[0, \varepsilon_{\mathrm{f}}\right]$, con $\varepsilon_{\mathrm{f}}>\varepsilon_{\mathrm{m}}$.

Para resolver los dos salarios, procedemos hacia atrás en el tiempo. Bajo los supuestos de que los salarios en el período $2, \mathrm{~W}_{2 \mathrm{i}}(\mathrm{i}=\mathrm{f}, \mathrm{m})$, son ofrecidos antes de que se realice $\omega_{2}$, que las empresas ya conocen $\delta$ en dicho período, que no hay renegociación salarial, y que el trabajador no ha abandonado en el período 1, escogerán $\mathrm{W}_{2 \mathrm{i}}$ de forma que maximice los beneficios esperados en el período $2, \Pi_{2}\left(\mathrm{~W}_{1 \mathrm{i}}\right)$, es decir, el producto de la probabilidad de no abandono en el período $1\left(\mathrm{~F}_{\mathrm{i}}\left(\mathrm{W}_{1 \mathrm{i}}\right)=\mathrm{W}_{1 \mathrm{i}} / \varepsilon_{\mathrm{i}}\right)$ y el beneficio en el período 2 , esto es

$$
\max _{W_{2 i}} \int_{0}^{W_{2 i}} \frac{W_{1 i}}{\varepsilon_{i}}\left(\gamma_{2} \delta-W_{2 i}\right) d F_{i}(\omega)=\max _{W_{2 i}} \frac{W_{1 i}}{\varepsilon_{i}}\left[\frac{\gamma_{2} \delta W_{2 i}}{\varepsilon_{i}}-\frac{W_{2 i}^{2}}{\varepsilon_{i}}\right], \quad i=f, m,
$$

donde la condición de primer orden (c.p.o.) con respecto a $\mathrm{W}_{2 \mathrm{i}}$ implica que el mismo salario será pagado en equilibrio a los trabajadores de ambos géneros con igual produc- 
tividad observada $\delta$, es decir $\mathrm{W}_{2 \mathrm{~m}} *=\mathrm{W}_{2 \mathrm{f}}{ }^{*}=\gamma_{2} \delta / 2^{12}$. Por consiguiente, definiendo el gap salarial entre géneros como $\left(\mathrm{W}_{2 \mathrm{~m}} *-\mathrm{W}_{2 \mathrm{f}}{ }^{*}\right)$, se cumple la siguiente proposición:

Proposición 1: El gap entre géneros en el período 2 es cero.

Sustituyendo $\mathrm{W}_{2}$ * en el término entre corchetes de (1), nótese que los beneficios esperados de la empresa al contratar hombres y mujeres son $\mathrm{W}_{1 \mathrm{~m}}\left(\gamma_{2} \delta\right)^{2} / 4 \varepsilon_{\mathrm{m}}{ }^{2}$ y $\mathrm{W}_{1 \mathrm{f}}$ $\left(\gamma_{2} \delta\right)^{2} / 4 \varepsilon_{\mathrm{f}}^{2}$, respectivamente. Por tanto, los beneficios en el período 2 (para $\delta$ conocido), $\Pi_{2}$, $\left(W_{i 1}\right)$, son función de los salarios pagados en el período inicial.

A continuación, habiendo elegido $\mathrm{W}_{2} *$, la empresa escoge el salario en el período $1, \mathrm{~W}_{1 \mathrm{i}}{ }^{*}$, para maximizar los beneficios esperados totales a la hora de contratar, esto es

$$
\max _{W_{1 i}} \int_{0}^{\varepsilon}\left\{\Pi_{2}\left(W_{i 1}\right)+\int_{0}^{W_{1 i}}\left(\gamma_{1 \delta}-W_{1 i}\right) d F_{i}(\omega)\right\} d G(\delta)
$$

Puesto que el término entre llaves en (2) es $\left(\gamma_{1} \delta W_{1 i}-W_{1 i}^{2}\right) / \varepsilon_{\mathrm{i}}$, la c.p.o. de (2) implica

$$
W_{1 i}^{*}=\frac{\gamma_{1} \tau}{4}+\frac{\gamma_{2}^{2} \tau^{2}}{24 \varepsilon_{1}}
$$

Dada la mayor probabilidad de abandono de las mujeres, el gap entre géneros en el período 1 es igual a $W_{1 m}^{*}-W_{1 f}^{*}=\frac{\gamma_{2}^{2} \tau^{2}}{24 \varepsilon_{m} \varepsilon_{f}}\left[\varepsilon_{f}-\varepsilon_{m}\right]>0$, de manera que se cumple el siguiente resultado: ${ }^{13}$

Proposición 2: El gap entre géneros en el período 1 es positivo. Adicionalmente, nótese que en tanto $\mathrm{W}_{2 \mathrm{i}}{ }^{*}>\mathrm{W}_{1 \mathrm{i}} *\left(\right.$ es decir, si $\left.\gamma_{2} \delta>\frac{\gamma_{1} \tau}{2}+\frac{\gamma_{2}^{2}}{12 \varepsilon_{i}} \tau^{2}\right)$, el re-
sultado previo implica que el salario aumenta con la experiencia ${ }^{14}$.

12 Esta expresión representa la media de la productividad del trabajador y su salario alternativo, que se supone nulo. La ponderación de $1 / 2$ en la media se debe al supuesto de una distribución uniforme en la ilustración del texto. Otras distribuciones darían lugar a una media ponderada con pesos diferentes.

13 Nótese que para los trabajadores de tipo $\mathrm{H}, \varepsilon_{m}=\varepsilon_{f}$ por lo que el gap salarial en el período 2 es cero. Por tanto el patrón de «techos de cristal» para este grupo de trabajadores debería explicarse mediante otras teorías, como las comentadas en la nota a p.p. 9, que no trataremos aquí.

14 Promediando la productividad, la desigualdad anterior se cumple para valores suficientemente elevados de $\varepsilon_{\mathrm{f}} \mathrm{y} \varepsilon_{\mathrm{m}}$. De nuevo, se pone de manifiesto que, aunque los resultados del modelo tendrían una interpretación más natural en términos de gaps salariales por edad o antigüedad (al haber dos períodos) que en términos de localización en la distribución salarial, se daría una interpretación similar en este último contexto si la edad y la antigüedad aumentarán monotónicamente a lo largo de la distribución (véase Cuadro 2 abajo). 
La intuición de ambas proposiciones es bastante simple. Dado que el segundo shock de desutilidad es desconocido cuando se ofrece $\mathrm{W}_{2 \mathrm{i}}$, lo mejor que puede hacer la empresa es igualar esta alternativa externa ofreciendo un salario igual a una fracción de la productividad observada $\gamma_{2} \delta$ que, en el caso de la distribución uniforme, es $\gamma_{2} \delta / 2$. Por tanto, la empresas obtienen un excedente en el período 2 de $\gamma_{2} \delta-W_{2} *=\gamma_{2} \delta / 2$. Nótese que, dada la c.p.o en el período 1, deberán pagar un salario por encima de $\gamma_{1} \delta / 4$ (i.e., el salario esperado condicional a no abandonar) en dicho período. Puesto que los beneficios esperados en el período 2 son mayores con los hombres (mas estables) que con las mujeres (menos estables), porque $\mathrm{W}_{1 \mathrm{~m}}{ }^{*}\left(\gamma_{2} \delta\right)^{2} / 4 \varepsilon_{\mathrm{m}}{ }^{2}>\mathrm{W}_{1 \mathrm{f}}{ }^{*}\left(\gamma_{2} \delta\right)^{2}$ $\left./ 4 \varepsilon_{\mathrm{f}}^{2}\right)$, las empresas pagarán un salario superior a aquellos en el período inicial. Ello explica: (i) por qué las mujeres reciben salarios inferiores a los hombres en el período 1 (trabajos de entrada en el mercado laboral), y (ii) por qué el perfil salarial es más plano que el perfil de productividad en el período 1.

¿Cuales son las implicaciones empíricas de estos resultados? Dado que los trabajos de entrada en el mercado laboral para los trabajadores del grupo L típicamente ofrecen salarios en la parte baja de la distribución, el resultado (i) sugiere que, para esta clase de trabajadores, el gap entre géneros es más elevado en la parte baja que en la parte alta de la distribución salarial, dando lugar al fenómeno de los suelos. A su vez, el resultado (ii) sugiere que en los trabajos de entrada al mercado laboral (el período 1 en el modelo), las mujeres de tipo L están estadísticamente discriminadas respecto a los hombres del mismo grupo, dado que el perfil salarial de las mujeres es más plano que el de su productividad. Sin embargo, en tanto las mujeres permanezcan en la empresa (i.e., tengan mayor antigüedad), la incertidumbre de los empresarios respecto a la posibilidad de abandono se reduce. Por tanto, los empresarios compensarán su anterior comportamiento discriminatorio aumentando el rendimiento de esta característica para las mujeres por encima de la de los hombres con igual productividad ${ }^{15}$.

\section{Regresiones cuantílicas: Metodología, datos y resultados}

\subsection{Metodología $(Q R)$}

De acuerdo con Koenker and Bassett (1978) y Buchinsky (1998a), el modelo de QR para una ecuación de salarios puede describirse como sigue. Sea $\left(\mathrm{w}_{\mathrm{i}}, \mathrm{x}_{\mathrm{i}}\right)$ una muestra aleatoria donde $\mathrm{w}_{\mathrm{i}}$ es el salario bruto por hora (en logs.) del individuo $\mathrm{i}, \mathrm{x}_{\mathrm{i}}$ es un vector $\mathrm{K} \times 1$ de regresores, $\mathrm{y} \mathrm{Q}_{\theta}\left(\mathrm{w}_{\mathrm{i}} \mid \mathrm{x}_{\mathrm{i}}\right)$ es el cuantil condicional $\theta^{\circ}$ de la distribución de $\mathrm{w}_{\mathrm{i}}$ dado $\mathrm{x}_{\mathrm{i}}$. Bajo el supuesto de una especificación lineal, el modelo puede definirse como

$$
\mathrm{w}_{\mathrm{i}}=\mathrm{x}_{\mathrm{i}} \beta_{\theta}+\mathrm{u}_{\theta \mathrm{i}}, \quad \mathrm{Q}_{\theta}\left(\mathrm{w}_{\mathrm{i}} \mid \mathrm{x}_{\mathrm{i}}\right)=\mathrm{x}_{\mathrm{i}}{ }^{\prime} \beta_{\theta}
$$

15 Esta explicación recuerda a aquella existente en la literatura sobre discriminación estadística relativa al proceso de aprendizaje, por parte de las empresas, de las habilidades del trabajador. Conforme el empresario aprende acerca del trabajador, a través de su mayor antigüedad en la empresa, el rendimiento de la educación (la señal) se reduce, mientras que los rendimientos de la experiencia y antigüedad aumentan (véase Farber y Gibbons, 1996). 
donde la distribución del término de error $\mathrm{u}_{\theta \mathrm{i}}, \mathrm{F}_{\mathrm{u} \theta}(\cdot)$, no necesita especificarse, suponiendo únicamente que $\mathrm{u}_{\theta \mathrm{i}}$ satisface $\mathrm{Q}_{\theta}\left(\mathrm{u}_{\theta \mathrm{i}} \mid \mathrm{x}_{\mathrm{i}}\right)=0$. El vector de estimadores de los coeficientes $\mathrm{QR}, \hat{\beta}_{\theta}$, se interpreta como el cambio marginal del cuantil condicional $\theta$ debido a un cambio marginal en en correspondiente elemento del vector de coeficientes de $\mathrm{x}$, que puede obtenerse mediante las técnicas de optimización descritas en Koenker and Bassett (1982).

Buchinsky (1998b) ha extendido el modelo (4) al caso en que se controla por el sesgo de selección inducido por el proceso de búsqueda de empleo. De la misma manera que en el modelo convencional de corrección paramétrica de la selección en la media condicional, habrán de considerarse dos salarios diferentes: (i) un salario de reserva, $\mathrm{w}_{\mathrm{i}}^{\mathrm{R}}$, que depende linealmente de un vector de características $\mathrm{z}_{\mathrm{i}}$, y (ii) una oferta salarial, $w_{i}{ }_{i}$, que también depende linealmente del vector $x_{i}$, tal que $\mathrm{Z}_{\mathrm{i}}$ contiene al menos un elemento que no está incluido en $\mathrm{x}_{\mathrm{i}}$. Puesto que la oferta salarial solamente es observada para los individuos que satisfacen $\mathrm{w}_{\mathrm{i}}{ }_{\mathrm{i}}>\mathrm{w}_{\mathrm{i}}^{\mathrm{R}}$, tenemos que $\mathrm{w}_{\mathrm{i}}=\mathrm{Dw}^{*}{ }_{\mathrm{i}}$ donde $\mathrm{D}$ es la función indicador $I\left(\mathrm{w}_{\mathrm{i}}^{*}>\mathrm{w}_{\mathrm{i}}^{\mathrm{R}}\right)$. Bajo condiciones débiles discutidas en Buchinsky (1998b), la probabilidad de trabajar $\mathrm{P}\left(\mathrm{w}_{\mathrm{i}}{ }_{\mathrm{i}}>\mathrm{w}_{\mathrm{i}}^{\mathrm{R}} / \mathrm{z}_{\mathrm{i}}\right)$ es una función de un índice conocido $g_{\mathrm{i}}\left(=\mathrm{z}_{\mathrm{i}}{ }^{\prime} \gamma\right)$, por lo que la ecuación de salarios observados es

$$
\mathrm{w}_{\mathrm{i}}=\mathrm{x}_{\mathrm{i}}{ }^{\prime} \beta_{\theta}+\mathrm{h}_{\theta}\left(\mathrm{g}_{\mathrm{i}}\right)+\varepsilon_{\theta \mathrm{i}}, \quad \mathrm{Q}_{\theta}\left(\varepsilon_{\theta \mathrm{i}} / \mathrm{x}_{\mathrm{i}}, \mathrm{D}=1\right)=0,(5)
$$

donde $h_{\theta}\left(g_{i}\right)$ se aproxima por una expansión en sucesivas potencias de la inversa de la ratio de Mills. Para poder estimar (5), los coeficientes desconocidos $\gamma$ se reemplazan por sus estimaciones obtenidas en una primera etapa a partir de la minimización de la distancia al cuadrado entre $\mathrm{D}_{\mathrm{i}} \mathrm{y}$ un kernel (no paramétrico) de la esperanza condicional $\mathrm{E}\left(\mathrm{D}_{\mathrm{i}} / \mathrm{z}_{\mathrm{i}}, \gamma\right)^{16}$.

\subsection{Datos y resultados}

Los datos se obtienen de la sexta ola, correspondiente a 1999 del PHOGUE que proporciona información armonizada para los países de la UE sobre salarios, empleo, horas de trabajo, educación, condición de inmigrante, estados civil y de salud, y otras variables socio-demográficas. La información procede de encuestas efectuadas a un panel fijo de hogares (70.000 en la UE y alrededor de 7.000 en España) desde 1994. Nuestra muestra está restringida a trabajadores a tiempo completo del grupo L en el segmento de edad 16-64, excluyendo a autónomos, estudiantes a tiempo completo y a aquellos que estaban realizando el servicio militar. Se dispone de 1.585 hombres y 726 mujeres. El Apéndice A contiene una descripción detallada de las variables usadas en las regresiones, mientras que el Cuadro 1 presenta un resumen de los estadís-

16 En la estimación reportada en la sección 3.2, el kernel no paramétrico es una normal truncada donde se utilizan los dos primeros términos de su expansión en potencias sucesivas. 
ticos descriptivos mas relevantes de la muestra ${ }^{17}$. Además, los correspondientes estadísticos para las mujeres no activas en el grupo L también se reportan en las dos últimas columnas de este Cuadro puesto que se utilizarán para corregir por selección no aleatoria. Se observa que los trabajadores masculinos del grupo L tienen una mayor experiencia laboral que las mujeres (4 años más) - donde la definición precisa de experiencia se ofrece posteriormente - tienen mayor antigüedad (1,8 años más) y son algo más viejos (1,7 años más). Las mujeres tienen una mayor presencia en empresas de menos de 20 empleados y trabajan con más frecuencia en el sector público. El gap salarial medio se sitúa en el 23\%. Por lo que se refiere a las mujeres no activas, tienen mayor edad que las que trabajan fuera del hogar (2,2 años más), una fracción mas alta tienen hijos pequeños y sus rentas no-laborales en el hogar es mas grande. Este último hecho parece indicar que las necesidades económicas de las mujeres trabajadoras son más altas.

Las dos primeras filas del Cuadro 2 presentan la evolución de experiencia y antigüiedad a lo largo de los cuantiles de la distribución salarial, confirmando que ambas aumentan monotónicamente conforme nos movemos hacia cuantiles más altos. Cuando se comparan los cuantiles $10^{\circ}$ y $90^{\circ}$, la experiencia y la antigüedad aumen$\tan$ en 32 y 20 años, respectivamente. Por tanto, para motivar los resultados en esta sección, nuestra hipótesis subyacente de que existe una relación entre los cuantiles salariales y las etapas de una carrera profesional parece sustentarse con esta base de datos. La tercera fila también reporta la fracción de contratos temporales por cuantiles. Se muestra que, pese a una tendencia decreciente, la diferencia en la antigüedad de los trabajadores por encima y por debajo de la mediana no puede explicarse exclusivamente porque el primer colectivo tenga contratos permanentes y el segundo contratos temporales ${ }^{18}$.

Se han estimado regresiones QR (reportadas para los cuantiles $10^{\circ}, 25^{\circ}, 50^{\circ}, 75^{\circ}$ y $90^{\circ}$ ) donde el salario bruto por hora (en logs.) se regresa sobre diferentes conjuntos de variables. La estimación robusta de heterocedasticidad en la media condicional también se ha llevado a cabo a efectos comparativos. Tal como es habitual en las ecuaciones salariales mincerianas, los controles son: experiencia y su cuadrado, experiencia interaccionada con edad de los hijos, antiguiedad en el actual puesto de tra-

17 Los estadísticos descriptivos de los hombres no activos pertenecientes al grupo L no se presentan pues su tasa de participación es alta (véase Cuadro A1). Además, los intentos por corregir los sesgos de selección no funcionaron: el término de selección siempre era no significativo y el resto de coeficientes de las características observables apenas cambiaban respecto a los obtenidos en regresiones sin corrección.

18 Pudiera uno preguntarse si la relación existente entre baja antigüedad y la tenencia de un contrato temporal pudiera afectar la validez de nuestra explicación basada en la hipótesis de discriminación estadística. Pensamos que no es el caso por cuatro razones. Primero, porque se controla por tipo de contrato en las regresiones salariales. Segundo, porque el coeficiente de un término de interacción entre antigüedad y modalidad contractual no es significativo. Tercero, porque, desde 1997, los contratos temporales con una duración máxima de 3 años fueron abolidos (véase Dolado et al., 2002). Finalmente, como se discutirá posteriormente, porque las descomposiciones contrafactuales simuladas en la sección 4 indican que la contratación temporal ejerce un papel mucho más reducido que la antigüedad a la hora de explicar el gap entre géneros. 
CUADRO 1

ESTADÍSTICOS DESCRIPTIVOS. TRABAJADORES GRUPO L. ESPAÑA (1999)

\begin{tabular}{|c|c|c|c|c|c|c|}
\hline & \multicolumn{2}{|c|}{ Hombres } & \multicolumn{2}{|c|}{ Mujeres } & \multicolumn{2}{|c|}{$\begin{array}{c}\text { Mujeres } \\
\text { (no participantes) }\end{array}$} \\
\hline & Media & Des. Est. & Media & Desv. Est. & Media & Desv. Est. \\
\hline No. Obs. & 1.585 & & 726 & & 998 & \\
\hline Edad & 37,89 & 11,31 & 36,19 & 11,33 & 38,81 & 8,39 \\
\hline Hijos $0-11$ & 0,30 & 0,46 & 0,21 & 0,41 & 0,53 & 0,50 \\
\hline $\begin{array}{l}\text { Hijos 12-16 } \\
\text { Grupo Edad }\end{array}$ & 0,16 & 0,37 & 0,17 & 0,38 &, 25 & 0,43 \\
\hline 17 a 24 & 0,13 & 0,33 & 0,18 & 0,38 & 0,14 & 0,35 \\
\hline 25 a 34 & 0,31 & 0,46 & 0,32 & 0,47 & 0,38 & 0,49 \\
\hline 35 a 44 & 0,25 & 0,43 & 0,23 & 0,42 & 0,43 & 0,50 \\
\hline$>45$ & 0,31 & 0,46 & 0,27 & 0,45 & 0,05 & 0,21 \\
\hline Casado & 0,68 & 0,47 & 0,53 & 0,50 & 0,73 & 0,44 \\
\hline Inmigrante & 0,04 & 0,07 & 0,02 & 0,08 & 0,01 & 0,09 \\
\hline Ed. Secundaria & 0,29 & 0,46 & 0,38 & 0,59 & 0,24 & 0,43 \\
\hline Horas Semanales & 42,64 & 6,19 & 40,36 & 5,74 & & \\
\hline Salario/Hora bruto & 1037 & 487 & 851 & 484 & & \\
\hline Log. (salario) & 6,86 & 0,41 & 6,63 & 0,47 & & \\
\hline Experiencia & 20,77 & 12,41 & 16,78 & 11,85 & & \\
\hline $\begin{array}{l}\text { Antigüedad } \\
\text { Ocupación }\end{array}$ & 8,28 & 7,87 & 6,48 & 7,23 & & \\
\hline $\mathrm{OC} 1$ & 0,02 & 0,14 & 0,01 & 0,11 & & \\
\hline $\mathrm{OC} 2$ & 0,00 & 0,00 & 0,00 & 0,00 & & \\
\hline OC3 & 0,00 & 0,03 & 0,00 & 0,04 & & \\
\hline OC4 & 0,00 & 0,06 & 0,00 & 0,07 & & \\
\hline OC5 & 0,02 & 0,13 & 0,02 & 0,14 & & \\
\hline OC6 & 0,05 & 0,21 & 0,06 & 0,24 & & \\
\hline OC7 & 0,07 & 0,25 & 0,18 & 0,38 & & \\
\hline OC8 & 0,08 & 0,28 & 0,18 & 0,38 & & \\
\hline OC9 & 0,04 & 0,20 & 0,13 & 0,33 & & \\
\hline OC10 & 0,02 & 0,15 & 0,01 & 0,10 & & \\
\hline OC11 & 0,22 & 0,42 & 0,08 & 0,27 & & \\
\hline OC12 & 0,10 & 0,30 & 0,01 & 0,10 & & \\
\hline OC13 & 0,20 & 0,40 & 0,06 & 0,24 & & \\
\hline OC14 & 0,05 & 0,21 & 0,20 & 0,40 & & \\
\hline OC15 & 0,13 & 0,33 & 0,06 & 0,24 & & \\
\hline Tamaño de Empresa & & & & & & \\
\hline 1-4 Empleados & 0,18 & 0,38 & 0,22 & 0,42 & & \\
\hline 5-19 Emp & 0,31 & 0,46 & 0,25 & 0,44 & & \\
\hline 20-49 Emp & 0,16 & 0,37 & 0,16 & 0,37 & & \\
\hline 50-99 Emp & 0,10 & 0,30 & 0,11 & 0,31 & & \\
\hline 100-499 Emp & 0,12 & 0,33 & 0,14 & 0,35 & & \\
\hline$>500 \mathrm{Emp}$ & 0,12 & 0,33 & 0,11 & 0,32 & & \\
\hline $\begin{array}{l}\text { Sector Público } \\
\text { Grado de supervisión }\end{array}$ & 0,13 & 0,34 & 0,18 & 0,38 & & \\
\hline Directivo & 0,05 & 0,23 & 0,03 & 0,16 & & \\
\hline Supervisor & 0,16 & 0,33 & 0,08 & 0,28 & & \\
\hline Sin responsabilidad & 0,79 & 0,41 & 0,89 & 0,31 & & \\
\hline Contrato permanente & 0,64 & 0,48 & 0,60 & 0,49 & & \\
\hline Padres 65-75 & 0,05 & 0,21 & 0,06 & 0,24 & 0,05 & 0,22 \\
\hline Padres $>76$ & 0,02 & 0,15 & 0,05 & 0,22 & 0,03 & 0,17 \\
\hline Renta No Laboral & 23.944 & 65.727 & 28.065 & 193.603 & 31.534 & 68.140 \\
\hline
\end{tabular}




\section{CUADRO 2}

EXPERIENCIA, ANTIGÜEDAD Y TEMPORALIDAD A LO LARGO DE LA DISTRIBUCIÓN SALARIAL (GRUPO L, ESPAÑA 1999)

\begin{tabular}{|l|r|r|r|r|r|r|}
\hline & Media & $\mathbf{- 1 0}$ & $\mathbf{-}=\mathbf{2 5}$ & $\mathbf{-}=\mathbf{5 0}$ & $\mathbf{-}=\mathbf{7 5}$ & $\mathbf{-}=\mathbf{9 0}$ \\
\hline$\quad$ Hombres (L) & & & & & & \\
Experiencia & 20,8 & 5,2 & 11,3 & 20,2 & 31,1 & 38,2 \\
Antigüedad & 8,3 & 0,5 & 1,2 & 3,4 & 15,0 & 20,3 \\
\% Contratos Temp. & 36,0 & 43,5 & 39,3 & 35,4 & 32,3 & 30,4 \\
\hline$\quad$ Mujeres (L) & & & & & & \\
Experiencia & 1609 & 2,2 & 7,2 & 15,3 & 26,2 & 34,3 \\
Antigüedad & 6,5 & 0,2 & 0,8 & 3,0 & 11,2 & 19,8 \\
\% Contratos Temp. & 40,0 & 45,2 & 40,1 & 36,3 & 33,1 & 30,9 \\
\hline
\end{tabular}

bajo, estado civil, edad de los hijos y educación secundaria. A esta altura, es importante señalar que el PHOGUE no ofrece información sobre la experiencia laboral real. Para subsanar este problema, lo mejor que puede hacerse con los datos disponibles es utilizar una medida de experiencia potencial computada como la «edad actual del individuo menos la edad a la que comenzó su primer empleo», que es una medida de la verdadera experiencia de las mujeres más certera que la convencional «edad menos años de escolaridad menos 6». Para mejorar algo más esta medida, siguiendo a Buchinsky (1998b), nuestra definición de experiencia potencial se interacciona con una variable ficticia de hijos dependientes menores de 16 años, suponiendo que la principal alternativa del trabajo asalariado es la cría y educación de los hijos. Para tener en cuenta el lado de la demanda del mercado laboral, también se incluyen variables ficticias regionales y de tamaño de la municipalidad. Otros controles son: tamaño de la empresa, condición de inmigrante, tipo de contrato (permanente o temporal), sector (privado o público), grado de supervisión y 15 variables ficticias ocupacionales. Estas últimas, también usadas por Albrecht et al. (2003), son probablemente endógenas, pero son útiles para explicar el gap entre géneros desde un punto de vista de ejercicio »contable», como señalan dichos autores ${ }^{19}$.

Como prueba preliminar, comenzamos el análisis mediante una regresión MCO conjunta (tipo pooling), tanto en la media como en los cuantiles antes mencionados, para la muestra conjunta de hombres y mujeres. Una variable ficticia de género (mujer=1) captura en qué medida permanece el gap sin explicar después de controlar por diferencias individuales en las características observadas, con los rendimientos restringidos a ser iguales para ambos géneros. Aunque los resultados de este ejercicio no

19 Desafortunadamente, el PHOGUE no proporciona información sobre el nivel educativo de los padres o su ocupación, que podrían ser instrumentos apropiados para corregir los sesgos de endogeneidad. 
se ofrecen para ahorrar espacio, resulta importante indicar que el coeficiente de la variable ficticia de género era negativo y estadísticamente significativo, disminuyendo en valor absoluto conforme nos movemos hacia cuantiles más altos en la distribución salarial. Sin embargo, estos resultados sólo pueden calificarse de tentativos ya que la hipótesis nula de igualdad de coeficientes de las variables para hombres y mujeres se rechazaba con un valor-p de 0.006 . Por tanto, resulta necesario estimar regresiones diferentes para cada género.

Seguidamente, para controlar la selección no aleatoria de los trabajadores de la muestra, se estiman separadamente regresiones QR con la corrección de Buchinsky descrita en la sección 3.1. Dado que la selección no aleatoria solo es significativa para las mujeres, se omiten los resultados de la ecuación de participación de los hombres. Los resultados de dicha ecuación para las mujeres del grupo L se presentan en el Cuadro 3a. La variable dependiente toma el valor 1 si la mujer trabaja y 0 si no lo hace, y las variables explicativas son: edad, número de hijos, edad de los mismos, edad de los padres que viven en el hogar, estado civil, condición de inmigrante y renta no-laboral del hogar. A efectos comparativos, la primera columna ofrece los coeficientes de una estimación probit, mientras que la segunda columna reporta las estimaciones de un modelo de single-index ${ }^{20}$. Los resultados obtenidos indican que las mujeres de mayor edad participan menos en el mercado laboral, como también afectan negativamente a la participación tener una renta no-laboral mayor, tener a los padres en casa o estar casada. Por el contrario, haber completado la educación secundaria o poseer la condición de inmigrante aumentan la probabilidad de trabajar.

Los Cuadros 3b (hombres) y $3 \mathrm{c}$ (mujeres) presentan los resultados de las QR estimadas separadamente para cada género. En primer lugar se ofrece la $\mathrm{QR}$ con corrección de selección para las mujeres, y sin corrección para los hombres. Adicionalmente, para chequear los efectos de la corrección, las estimaciones sin la misma para las mujeres se exponen también en el Cuadro 3d. Comenzando con la comparación entre hombres y mujeres, los Cuadros $3 b$ y $3 c$ revelan que el coeficiente estimado para edad/experiencia es más grande en el caso de los hombres que en el de las mujeres y que dicha diferencia se agranda conforme se avanza hacia arriba en la distribución. Además, haber completado la educación secundaria presenta un rendimiento de mercado mayor para las mujeres en los cuantiles bajos, como también es el caso de trabajar en el sector público o tener un contrato permanente. El coeficiente de la variable estar casado es mayor para los hombres, especialmente en los cuantiles bajos. Sin embargo, el resultado más interesante es que los coeficientes de la antigüedad son más altos para las mujeres que para los hombres en los cuantiles bajos pero convergen al mismo valor conforme nos movemos hacia arriba en la distribución. Nótese que este patrón de diferencias puede interpretarse fácilmente en términos de nuestra ex-

20 Tal como se explica en Buchinsky (1998b, pg.7), la constante y el coeficiente de una de las variables continuas (p. ej., la edad) no están identificadas en un modelo de single-index Por tanto, se normalizan sus valores igualándolos a los obtenidos en el modelo probit de manera que los resultados sean comparables. 


\section{CUADRO 3a}

\section{ESTIMACIÓN DE LA PROBABILIDAD DE TRABAJAR (GRUPO L, ESPAÑA (1999)}

\begin{tabular}{|c|c|c|}
\hline MUJERES & Probit & $\begin{array}{l}\text { Single } \\
\text {-index }\end{array}$ \\
\hline Constante & $\begin{array}{c}-0,334 * * * \\
(0,004)\end{array}$ & $\begin{array}{c}-0,334 \\
(.) \dagger\end{array}$ \\
\hline Edad & $\begin{array}{c}-0,045 * * * \\
(0,002)\end{array}$ & $\begin{array}{c}-0,045 \\
(.) \dagger\end{array}$ \\
\hline No. de Hijos & $\begin{array}{c}-0,053 * * * \\
(0,016)\end{array}$ & $\begin{array}{c}-0,060 * * * \\
(0,024)\end{array}$ \\
\hline Hijos 0-11 & $\begin{array}{c}-0,085 * * * \\
(0,024)\end{array}$ & $\begin{array}{c}-0,078 \text { *** } \\
(0,031)\end{array}$ \\
\hline Padres $>65$ & $\begin{array}{c}-0,074 * * \\
(0,035)\end{array}$ & $\begin{array}{c}-0,098 * * \\
(0,048)\end{array}$ \\
\hline Ed. Secundaria & $\begin{array}{c}0,067 * * * \\
(0,012)\end{array}$ & $\begin{array}{c}0,074 * * * \\
(0,014)\end{array}$ \\
\hline Casada & $\begin{array}{c}-0,092 * * * \\
(0,007)\end{array}$ & $\begin{array}{c}-0,083 * * * \\
(0,011)\end{array}$ \\
\hline Inmigrante & $\begin{array}{c}0,034 * * \\
(0,016)\end{array}$ & $\begin{array}{c}0,045^{* *} \\
(0,021)\end{array}$ \\
\hline Renta No Laboral / 100 & $\begin{array}{c}-0,056 * * * \\
(0,009)\end{array}$ & $\begin{array}{c}-0,046 * * * \\
(0,012)\end{array}$ \\
\hline No. Obs. & 1.724 & 1.724 \\
\hline
\end{tabular}

Nota: $†$ La constante y el coeficiente de la edad están Normalizados en el modelo single-index.

$* * *, * *, *$ representan significativo al 99, 95 y 90\% respectivamente. Desviación Estándar en paréntesis.

plicación basada en la discriminación estadística. En efecto, la estabilidad de las mujeres de tipo L en sus empleos es un suceso incierto para los empresarios, particularmente en las fases iniciales de sus carreras profesionales, las cuales, de acuerdo con la evidencia en el Cuadro 2, es probable que correspondan a la parte baja de la distribución salarial. Por tanto, el rendimiento adicional de una mayor antigüedad en el puesto de trabajo (i.e., una reducción de la incertidumbre de los empresarios) debería ser más elevado para ellas que para los hombres, considerados «mas estables».

La comparación de los resultados en los Cuadros $3 \mathrm{c}$ y $3 \mathrm{~d}$ nos permite chequear los efectos de la corrección de los posibles sesgos de selección en la muestra de las mujeres. Lo primero que llama la atención es que tanto el tamaño del término constante como los de los coeficientes de diversas variables ficticias (con valor 1 para las mujeres) en el Cuadro $3 \mathrm{c}$ caen relativamente a los reportados en el Cuadro $3 \mathrm{~d}$, implicando un gap salarial medio del $22,6 \%$ en vez del $19,7 \%$ que se obtiene sin corrección. Además, esta reducción es mucho mayor en la parte baja que en la parte alta de la distribución. Finalmente, el perfil decreciente del coeficien- 
te estimado de la antigüedad tiene una pendiente negativa mucho más pronunciada en la ecuación con corrección que en la ecuación sin corrección. En resumen, la corrección por selección agudiza el fenómeno de los suelos. En consecuencia, este resultado descarta la explicación alternativa del patrón de suelos como un fenómeno puro de selección muestral, de acuerdo con el cual las mujeres cuyos salarios se sitúan en las partes media y alta de la distribución constituyen una

\section{CUADRO 3b}

MCO Y QR (GRUPO L. ESPAÑA 1999)

\begin{tabular}{|c|c|c|c|c|c|c|}
\hline \multicolumn{7}{|c|}{ Variable dependiente: (Ln.) salario bruto por hora } \\
\hline HOMBRES & MCO & $=10$ & _ $=25$ & $\_=50$ & _ $=75$ & _ $=90$ \\
\hline Constante & $\begin{array}{c}1,385 * * * \\
(0,047)\end{array}$ & $\begin{array}{c}1,0157 * * * \\
(0,062)\end{array}$ & $\begin{array}{c}1,295^{* * * *} \\
(0,063)\end{array}$ & $\begin{array}{c}1,379 * * * \\
(0,058)\end{array}$ & $\begin{array}{c}1,459 * * * \\
(0,064)\end{array}$ & $\begin{array}{c}1,557 * * * \\
(0,069)\end{array}$ \\
\hline Experiencia & $\begin{array}{c}0,013 * * * \\
(0,003)\end{array}$ & $\begin{array}{c}0,017 * * * \\
(0,006)\end{array}$ & $\begin{array}{c}0,010^{* * * *} \\
(0,003)\end{array}$ & $\begin{array}{c}0,010 * * * \\
(0,003)\end{array}$ & $\begin{array}{l}0,008^{*} \\
(0,004)\end{array}$ & $\begin{array}{c}0,008 \\
(0,005)\end{array}$ \\
\hline Experiencia2 & $\begin{array}{c}-0,0002 * * * \\
(0,00005)\end{array}$ & $\begin{array}{c}-0,0003^{* *} \\
(0,0001)\end{array}$ & $\begin{array}{c}-0,002 * * * \\
(0,0007)\end{array}$ & $\begin{array}{c}-0,002 * * * \\
(0,0007)\end{array}$ & $\begin{array}{c}-0,0001 \\
(0,00009)\end{array}$ & $\begin{array}{l}-0,0001 \\
(0,0001)\end{array}$ \\
\hline Exp*No. hijos & $\begin{array}{c}-0,002 \text { ** } \\
(0,0008)\end{array}$ & $\begin{array}{c}-0,003^{*} \\
(0,0001)\end{array}$ & $\begin{array}{l}-0,002 \\
(0,001)\end{array}$ & $\begin{array}{c}-0,001 \\
(0,0009)\end{array}$ & $\begin{array}{c}-0,001 \\
(0,0009)\end{array}$ & $\begin{array}{l}-0,001 \\
(0,001)\end{array}$ \\
\hline Ed. Secundaria & $\begin{array}{c}0,060 * * * \\
(0,020)\end{array}$ & $\begin{array}{l}0,081^{* *} \\
(0,040)\end{array}$ & $\begin{array}{c}0,060 * * \\
(0,027)\end{array}$ & $\begin{array}{c}0,055^{* * *} \\
(0,019)\end{array}$ & $\begin{array}{c}0,025 \\
(0,024)\end{array}$ & $\begin{array}{c}0,023 \\
(0,035)\end{array}$ \\
\hline Inmigrante & $\begin{array}{l}-0,143 \\
(0,144)\end{array}$ & $\begin{array}{l}-0,192 \\
(0,130)\end{array}$ & $\begin{array}{l}-0,222 \\
(0,150)\end{array}$ & $\begin{array}{l}-0,244 \\
(0,165)\end{array}$ & $\begin{array}{c}0,005 \\
(0,270)\end{array}$ & $\begin{array}{c}0,151 \\
(0,265)\end{array}$ \\
\hline Sector Público & $\begin{array}{c}0,020 \\
(0,030)\end{array}$ & $\begin{array}{c}0,006 \\
(0,049)\end{array}$ & $\begin{array}{c}0,033 \\
(0,038)\end{array}$ & $\begin{array}{c}0,032 \\
(0,027)\end{array}$ & $\begin{array}{c}0,036 \\
(0,040)\end{array}$ & $\begin{array}{c}0,054 \\
(0,047)\end{array}$ \\
\hline Grado Supervisión & $\begin{array}{c}0,065^{* * *} \\
(0,022)\end{array}$ & $\begin{array}{c}0,105^{* *} \\
(0,048)\end{array}$ & $\begin{array}{c}0,061^{* *} \\
(0,030)\end{array}$ & $\begin{array}{c}0,028 \\
(0,026)\end{array}$ & $\begin{array}{c}0,037 \\
(0,027)\end{array}$ & $\begin{array}{c}0,073^{* *} \\
(0,037)\end{array}$ \\
\hline Directivo & $\begin{array}{c}0,161 * * * \\
(0,040)\end{array}$ & $\begin{array}{c}0,193 * * * \\
(0,073)\end{array}$ & $\begin{array}{c}0,113^{* *} \\
(0,051)\end{array}$ & $\begin{array}{c}0,166 * * * \\
(0,050)\end{array}$ & $\begin{array}{c}0,135^{* *} \\
(0,068)\end{array}$ & $\begin{array}{l}0,104^{*} \\
(0,059)\end{array}$ \\
\hline Supervisor & $\begin{array}{c}0,089 * * * \\
(0,023)\end{array}$ & $\begin{array}{c}0,083 * * \\
(0,035)\end{array}$ & $\begin{array}{c}0,080^{* * * *} \\
(0,028)\end{array}$ & $\begin{array}{c}0,096 * * * * \\
(0,031)\end{array}$ & $\begin{array}{c}0,092 * * * \\
(0,032)\end{array}$ & $\begin{array}{c}0,074 * * \\
(0,035)\end{array}$ \\
\hline Antigüedad & $\begin{array}{c}0,012 * * * \\
(0,003)\end{array}$ & $\begin{array}{c}0,0015^{* * *} \\
(0,004)\end{array}$ & $\begin{array}{c}0,014 * * * \\
(0,004)\end{array}$ & $\begin{array}{c}0,012^{* *} \\
(0,005)\end{array}$ & $\begin{array}{l}0,009 * \\
(0,006)\end{array}$ & $\begin{array}{l}0,010^{*} \\
(0,006)\end{array}$ \\
\hline Casado & $\begin{array}{c}0,079 * * * \\
(0,021)\end{array}$ & $\begin{array}{c}0,123 * * * \\
(0,038)\end{array}$ & $\begin{array}{c}0,0083^{* * *} * \\
(0,025)\end{array}$ & $\begin{array}{c}0,070 * * * \\
(0,027)\end{array}$ & $\begin{array}{c}0,073 * * \\
(0,030)\end{array}$ & $\begin{array}{c}0,077 * * \\
(0,033)\end{array}$ \\
\hline $\mathrm{N}^{\circ}$ Obs. & 1.585 & 1.585 & 1.585 & 1.585 & 1.585 & 1.585 \\
\hline R2 & 0,333 & 0,237 & 0,250 & 0,282 & 0,327 & 0,350 \\
\hline
\end{tabular}

Nota: $* * *, * * *$ representan significativo al 99, 95 y 90\% respectivamente. Desviación Estándar en paréntesis. Se incluyen también variables ficticias de región, tamaño de empresa y municipalidad y de ocupaciones. Grupo omitido (referencia): asalariados en el sector privado en empresas de menos de 5 trabajadores, solteros, con educación primaria, en empleos no cualificados en servicios y comercio (OC14). 


\section{CUADRO $3 \mathrm{c}$}

MCO Y QR (CON CORRECIÓN POR SESGOS DE SELECCIÓN, GRUPO L. ESPAÑA 1999)

\begin{tabular}{|c|c|c|c|c|c|c|}
\hline \multicolumn{7}{|c|}{ Variable dependiente: (Ln.) salario bruto por hora } \\
\hline MUJERES & MCO & $=\mathbf{1 0}$ & $-=25$ & $-\mathbf{= 5 0}$ & $-=75$ & $-=90$ \\
\hline Constante & $\begin{array}{l}1,267 * * * \\
(0,0481)\end{array}$ & $\begin{array}{c}1,087 * * * \\
(0,076)\end{array}$ & $\begin{array}{c}1,252 * * * \\
(0,070)\end{array}$ & $\begin{array}{c}1,263 * * * \\
(0,073)\end{array}$ & $\begin{array}{c}1,438 * * * \\
(0,069)\end{array}$ & $\begin{array}{c}1,546^{* * * *} \\
(0,069)\end{array}$ \\
\hline Experiencia & $\begin{array}{c}0,006 * * \\
(0,003)\end{array}$ & $\begin{array}{c}0,006 \\
(0,007)\end{array}$ & $\begin{array}{c}0,007 \\
(0,006)\end{array}$ & $\begin{array}{l}0,008^{*} \\
(0,005)\end{array}$ & $\begin{array}{l}0,009 * \\
(0,005)\end{array}$ & $\begin{array}{l}0,009 * \\
(0,006)\end{array}$ \\
\hline Experiencia2 & $\begin{array}{l}-0,0001 \\
(0,0007)\end{array}$ & $\begin{array}{c}0,0001 \\
(0,0002)\end{array}$ & $\begin{array}{l}-0,0001 \\
(0,0001)\end{array}$ & $\begin{array}{l}-0,0001 \\
(0,0001)\end{array}$ & $\begin{array}{l}-0,0001 \\
(0,0001)\end{array}$ & $\begin{array}{l}-0,0001 \\
(0,0001)\end{array}$ \\
\hline Exp* $\mathrm{N}^{\mathrm{o}}$ hijos & $\begin{array}{c}-0,003 * * * \\
(0,001)\end{array}$ & $\begin{array}{c}0,0007 \\
(0,005\end{array}$ & $\begin{array}{c}-0,006 * * * \\
(0,002)\end{array}$ & $\begin{array}{c}-0,004^{*} \\
(0,002)\end{array}$ & $\begin{array}{c}-0,003 * \\
(0,002)\end{array}$ & $\begin{array}{l}-0,002 \\
(0,002)\end{array}$ \\
\hline Ed. Secundaria & $\begin{array}{c}0,104 * * * \\
(0,033)\end{array}$ & $\begin{array}{c}0,096 * * * \\
(0,50)\end{array}$ & $\begin{array}{c}0,081 * * \\
(0,039)\end{array}$ & $\begin{array}{l}0,051^{*} \\
(0,030)\end{array}$ & $\begin{array}{c}0,026 \\
(0,039)\end{array}$ & $\begin{array}{c}0,025 \\
(0,044)\end{array}$ \\
\hline Inmigrante & $\begin{array}{c}-0,512 * * * \\
(0,066)\end{array}$ & $\begin{array}{c}-0,283^{* *} \\
(0,137)\end{array}$ & $\begin{array}{c}-0,387 * * * \\
(0,103)\end{array}$ & $\begin{array}{c}-0,556 * * * \\
(0,131)\end{array}$ & $\begin{array}{c}-0,597 * * * \\
(0,132)\end{array}$ & $\begin{array}{c}-0,606^{* * *} * \\
(0,142)\end{array}$ \\
\hline Sector Público & $\begin{array}{c}0,097 * * * \\
(0,036)\end{array}$ & $\begin{array}{c}0,150 * * \\
(0,073)\end{array}$ & $\begin{array}{c}0,134 * * \\
(0,066)\end{array}$ & $\begin{array}{c}0,066^{* *} \\
(0,031)\end{array}$ & $\begin{array}{l}0,067^{*} \\
(0,040)\end{array}$ & $\begin{array}{l}0,068^{*} \\
(0,042)\end{array}$ \\
\hline $\begin{array}{l}\text { Contrato permanente } \\
\text { Grado Supervisión }\end{array}$ & $\begin{array}{c}0,098 * * * \\
(0,033)\end{array}$ & $\begin{array}{c}0,194 * * * \\
(0,062)\end{array}$ & $\begin{array}{c}0,160 * * * \\
(0,060)\end{array}$ & $\begin{array}{c}0,111^{* *} \\
(0,048)\end{array}$ & $\begin{array}{l}0,092 * \\
(0,049)\end{array}$ & $\begin{array}{c}0,120 * * \\
(0,051)\end{array}$ \\
\hline Directivo & $\begin{array}{l}-0,041 \\
(0,108)\end{array}$ & $\begin{array}{l}-0,007 \\
(0,197)\end{array}$ & $\begin{array}{c}0,013 \\
(0,129)\end{array}$ & $\begin{array}{c}0,083 \\
(0,131)\end{array}$ & $\begin{array}{c}0,085 \\
(0,179)\end{array}$ & $\begin{array}{c}0,062 \\
(0,173)\end{array}$ \\
\hline Supervisor & $\begin{array}{l}0,069 * \\
(0,041)\end{array}$ & $\begin{array}{c}0,075 \\
(0,081)\end{array}$ & $\begin{array}{c}0,073 \\
(0,061)\end{array}$ & $\begin{array}{c}0,086 * * \\
(0,043)\end{array}$ & $\begin{array}{c}0,065 \\
(0,061)\end{array}$ & $\begin{array}{c}0,063 \\
(0,072)\end{array}$ \\
\hline Antigüedad & $\begin{array}{c}0,024 * * * \\
(0,003)\end{array}$ & $\begin{array}{c}0,033 * * * \\
(0,006)\end{array}$ & $\begin{array}{c}0,029 * * * \\
(0,006)\end{array}$ & $\begin{array}{c}0,013 * * * \\
(0,005)\end{array}$ & $\begin{array}{c}0,009 \\
(0,007)\end{array}$ & $\begin{array}{c}0,011 \\
(0,009)\end{array}$ \\
\hline Casada & $\begin{array}{c}0,060 * * \\
(0,024)\end{array}$ & $\begin{array}{c}0,064^{*} \\
(0,04)\end{array}$ & $\begin{array}{l}0,067^{*} \\
(0,043)\end{array}$ & $\begin{array}{c}0,093 * * * \\
(0,032)\end{array}$ & $\begin{array}{c}0,022 \\
(0,033)\end{array}$ & $\begin{array}{c}0,086 \\
(0,044)\end{array}$ \\
\hline $\begin{array}{l}\mathrm{N}^{\mathrm{o}} \text { Obs. } \\
\mathrm{R} 2\end{array}$ & $\begin{array}{c}726 \\
0,3323\end{array}$ & $\begin{array}{c}726 \\
0,385\end{array}$ & $\begin{array}{c}726 \\
0,379\end{array}$ & $\begin{array}{c}726 \\
0,396\end{array}$ & $\begin{array}{c}726 \\
0,456\end{array}$ & $\begin{array}{c}726 \\
0,471\end{array}$ \\
\hline
\end{tabular}

Nota: ***,**,* representan significativo al 99, 95 y 90\% respectivamente. Desviación Estándar en paréntesis. Se incluyen también variables ficticias de región, tamaño de empresa y municipalidad y de ocupaciones. Grupo omitido (referencia): asalariados en el sector privado en empresas de menos de 5 trabajadores, solteros, con educación primaria, en empleos no cualificados en servicios y comercio (OC14).

muestra favorablemente seleccionada en términos de sus características productivas. Por el contrario, apoya el hecho de que las mujeres con salarios bajos son una muestra favorablemente seleccionada, exacerbando de esta forma el perfil decreciente de los suelos. Adicionalmente, las diferencias estimadas en los coeficientes de la antigüedad por género apoyan a nuestra explicación basada en la hipótesis de discriminación estadística. 


\section{CUADRO 3D}

MCO Y QR (SIN CORRECIÓN POR SESGOS DE SELECCIÓN,
GRUPO L. ESPAÑA 1999)

\begin{tabular}{|c|c|c|c|c|c|c|}
\hline \multicolumn{7}{|c|}{ Variable dependiente: (Ln.) salario bruto por hora } \\
\hline MUJERES & MCO & _=10 & $-=25$ & $-=50$ & $-=75$ & $-=90$ \\
\hline Constante & $\begin{array}{c}10308^{* * *} \\
(0,051)\end{array}$ & $\begin{array}{c}10118 * * * \\
(0,082)\end{array}$ & $\begin{array}{c}1,295 * * * \\
(0,074)\end{array}$ & $\begin{array}{c}1,313 * * * \\
(0,078)\end{array}$ & $\begin{array}{c}10447 * * * \\
(0,074)\end{array}$ & $\begin{array}{c}10555^{* * *} \\
(0,073)\end{array}$ \\
\hline Experiencia & $\begin{array}{l}0,007 * \\
(0,004)\end{array}$ & $\begin{array}{c}-0,00001 \\
(0,010)\end{array}$ & $\begin{array}{c}0,009 \\
(0,006)\end{array}$ & $\begin{array}{c}0,008 \\
(0,005)\end{array}$ & $\begin{array}{c}0,006 \\
(0,006)\end{array}$ & $\begin{array}{c}0,006 \\
(0,007)\end{array}$ \\
\hline Experiencia2 & $\begin{array}{c}-0,0001 \\
(0,00009)\end{array}$ & $\begin{array}{c}0,0001 \\
(0,0002)\end{array}$ & $\begin{array}{l}-0,0001 \\
(0,0001)\end{array}$ & $\begin{array}{l}-0,0001 \\
(0,0001)\end{array}$ & $\begin{array}{l}-0,0001 \\
(0,0001)\end{array}$ & $\begin{array}{l}-0,0001 \\
(0,0001)\end{array}$ \\
\hline Exp*N ${ }^{o}$ hijos & $\begin{array}{l}-0,002 \\
(0,001)\end{array}$ & $\begin{array}{c}0,00004 \\
(0,004)\end{array}$ & $\begin{array}{c}-0,004 * * \\
(0,002)\end{array}$ & $\begin{array}{c}-0,003 * \\
(0,002)\end{array}$ & $\begin{array}{c}0,002 \\
(0,002)\end{array}$ & $\begin{array}{c}0,002 \\
(0,002)\end{array}$ \\
\hline Ed. Secundaria & $\begin{array}{c}0,113 * * * \\
(0,033)\end{array}$ & $\begin{array}{l}0,103^{*} \\
(0,059)\end{array}$ & $\begin{array}{l}0,077 * \\
(0,043)\end{array}$ & $\begin{array}{l}0,059^{*} \\
(0,040)\end{array}$ & $\begin{array}{c}0,028 * * \\
(0,038)\end{array}$ & $\begin{array}{c}0,027 \\
(0,040)\end{array}$ \\
\hline Inmigrante & $\begin{array}{c}-0,479 * * * \\
(0,073)\end{array}$ & $\begin{array}{c}-0,249 * \\
(0,140)\end{array}$ & $\begin{array}{c}-0,358 * * * \\
(0,112)\end{array}$ & $\begin{array}{c}-0,528 * * * \\
(0,148)\end{array}$ & $\begin{array}{c}-0,584 * * * \\
(0,144)\end{array}$ & $\begin{array}{c}-0,668 * * * \\
(0,173)\end{array}$ \\
\hline Sector Público & $\begin{array}{c}0,108 * * * \\
(0,039)\end{array}$ & $\begin{array}{c}0,150 * * \\
(0,073)\end{array}$ & $\begin{array}{c}0,134 * * \\
(0,066)\end{array}$ & $\begin{array}{c}0,066 \\
(0,051)\end{array}$ & $\begin{array}{c}0,067 \\
(0,060)\end{array}$ & $\begin{array}{c}0,066 \\
(0,072\end{array}$ \\
\hline $\begin{array}{l}\text { Contrato permanente } \\
\text { Grado Supervisión }\end{array}$ & $\begin{array}{c}0,121^{* * *} * \\
(0,035)\end{array}$ & $\begin{array}{c}0,194 * * * \\
(0,0162)\end{array}$ & $\begin{array}{c}0,160 * * * \\
(0,060)\end{array}$ & $\begin{array}{c}0,111^{* *} \\
(0,048)\end{array}$ & $\begin{array}{c}0,090 * * \\
(0,045)\end{array}$ & $\begin{array}{c}0,104 * * \\
(0,048)\end{array}$ \\
\hline Directivo & $\begin{array}{l}-0,050 \\
(0,129)\end{array}$ & $\begin{array}{c}0,018 \\
(0,210)\end{array}$ & $\begin{array}{c}0,017 \\
(0,138)\end{array}$ & $\begin{array}{l}-0,150 \\
(0,127)\end{array}$ & $\begin{array}{c}0,079 \\
(0,184)\end{array}$ & $\begin{array}{c}0,060 \\
(0,176)\end{array}$ \\
\hline Supervisor & $\begin{array}{c}0,075^{*} \\
(0,045)\end{array}$ & $\begin{array}{c}0,081 \\
(0,088)\end{array}$ & $\begin{array}{c}0,094 \\
(0,061)\end{array}$ & $\begin{array}{c}0,096 * * \\
(0,049)\end{array}$ & $\begin{array}{c}0,070 \\
(0,066)\end{array}$ & $\begin{array}{c}0,67 \\
(0,078)\end{array}$ \\
\hline Antigüedad & $\begin{array}{c}0,021 * * * \\
(0,004)\end{array}$ & $\begin{array}{c}0,027 * * * \\
(0,005)\end{array}$ & $\begin{array}{c}0,024 * * * \\
(0,006)\end{array}$ & $\begin{array}{c}0,016 * * * \\
(0,006)\end{array}$ & $\begin{array}{c}0,013 * * \\
(0,007)\end{array}$ & $\begin{array}{c}0,010 \\
(0,010)\end{array}$ \\
\hline Casada & $\begin{array}{c}0,065^{* *} \\
(0,027)\end{array}$ & $\begin{array}{c}0,040 \\
(0,050)\end{array}$ & $\begin{array}{l}0,071 * \\
(0,043)\end{array}$ & $\begin{array}{c}0,093 * * * \\
(0,032)\end{array}$ & $\begin{array}{c}0,022 \\
(0,033)\end{array}$ & $\begin{array}{c}0,086 \\
(0,044)\end{array}$ \\
\hline $\begin{array}{l}\mathrm{N}^{\circ} \text { Obs. } \\
\mathrm{R} 2\end{array}$ & $\begin{array}{c}726 \\
0,308\end{array}$ & $\begin{array}{c}726 \\
0,372\end{array}$ & $\begin{array}{c}726 \\
0,372\end{array}$ & $\begin{array}{c}726 \\
0,385\end{array}$ & $\begin{array}{c}726 \\
0,421\end{array}$ & $\begin{array}{c}726 \\
0,462\end{array}$ \\
\hline
\end{tabular}

Nota: ***,**,* representan significativo al 99, 95 y 90\% respectivamente. Desviación Estándar en paréntesis. Se incluyen también variables ficticias de región, tamaño de empresa y municipalidad y de ocupaciones. Grupo omitido (referencia): asalariados en el sector privado en empresas de menos de 5 trabajadores, solteros, con educación primaria, en empleos no cualificados en servicios y comercio (OC14).

Globalmente, la evidencia presentada hasta ahora indica que: (i) los rendimientos de las características productivas observables difieren por género, (ii) estas diferencias varían a lo largo de la distribución, y (iii) la corrección de los sesgos de selección agudiza el fenómeno de los suelos. El siguiente paso es investigar la importancia de los componentes inobservables a la hora de explicar el gap entre géneros, con el fin de contrastar nuestra hipótesis de discriminación estadística. 


\section{Decomposición de los gaps por género}

\subsection{Metodología (descomposición MM)}

Tal como es bien conocido, la descomposición de Oaxaca-Blinder se usa para comparar los salarios medios observados de hombres y mujeres con los contrafactuales. En concreto, denotando a los rendimientos de las características de hombres y mujeres como $\beta_{\mathrm{f}}$ and $\beta_{\mathrm{m}} \mathrm{y}$ sus características como $\mathrm{x}_{\mathrm{f}}$ and $\mathrm{x}_{\mathrm{m}}$, esta descomposición implica que $\mathrm{E}\left(\mathrm{w}^{\mathrm{m}}\right)-\mathrm{E}\left(\mathrm{w}^{\mathrm{f}}\right)=\left(\mathrm{E}\left(\mathrm{x}^{\mathrm{m}}\right)-\mathrm{E}\left(\mathrm{x}^{\mathrm{f}}\right)\right)^{\prime} \beta^{\mathrm{m}}+\mathrm{E}\left(\mathrm{x}^{\mathrm{f}}\right)^{\prime}\left(\beta^{\mathrm{m}}-\beta^{f}\right)$. $\mathrm{El}$ primer término mide las diferencias en los salarios medios debidos a diferente dotación de características, mientras que el segundo término captura el diferencial debido a diferentes rendimientos de dichas características ${ }^{21}$.

Una generalización de la descomposición de Oaxaca-Blinder al contexto de QR ha sido propuesta por Machado y Mata (2005; en adelante MM) mediante el uso de métodos de Monte Carlo. Esta descomposición se basa en la construcción de una distribución contrafactual de $w^{f}$ que representa la distribución de salarios femeninos que hubiera prevalecido si las mujeres estuvieran dotadas con sus propias características pero cuyo rendimiento en el mercado laboral fuera el de los hombres. Se denota a esta distribución contrafactual como $F\left(\widetilde{w}^{f} / x^{f}, \hat{\beta}_{\theta}^{m}\right)$, donde $\widetilde{w}^{f}$ son los valores generados de $w^{f}$, y $\hat{\beta}_{\theta}^{m}$ son los coeficientes (masculinos) de la QR.

Los pasos en el algoritmo MM con el fin de construir $F\left(\widetilde{w}^{f} / x^{f}, \hat{\beta}_{\theta}^{m}\right)$, puede resumirse como sigue.

- Para cada cuantil $\theta=0,01,0,02, . ., 0,99$, se estima el vector de coeficientes $\hat{\beta}_{\theta}^{m}$ con QR usando la base de datos de los hombres.

- Para cada $\theta$, se extrae una realización (con reemplazamiento) de la base de datos de las mujeres con el fin de generar el valor ajustado del salario femenino, $\widetilde{w}^{f}(\theta)=\hat{\beta}_{\theta}^{m^{\prime}} x^{f}$. Ello genera $\mathrm{N}^{\mathrm{f}}$ valores ajustados, donde $\mathrm{N}^{\mathrm{f}}$ es el tamaño de la muestra de mujeres. A continuación, se genera de manera aleatoria $s=100$ de los elementos de $\widetilde{w}^{f}(\theta)$ para cada $\theta$ y se disponen en un vector de elementos 99x100, $\widetilde{w}^{f}$. La función de distribución empírica de estos valores es la distribución contrafactual estimada, esto es, lo que las mujeres hubieran obtenido si fueran pagadas como los hombres.

- Se compara la distribución contrafactual con las distribuciones empíricas de los salarios de hombres y mujeres cuyos cuantiles $\theta$ están definidos por $w^{m}(\theta)$ y $w^{f}$ $(\theta)$, respectivamente. El gap entre géneros en el cuantil $\theta$ puede descomponerse como: $w^{m}(\theta)-w^{f}(\theta)=\left[w^{m}(\theta)-\widetilde{w}^{f}(\theta)\right]+\left[\tilde{w}^{f}(\theta)-w^{f}(\theta)\right]$. El primer término en corchetes corresponde al efecto características puesto que mide la contribución de las diferentes variables explicativas al gap salarial en el cuantil $\theta$.

21 Las diferencias en las características observables se evalúan típicamente en la descomposición usando los rendimientos masculinos, bajo el supuesto de que estos últimos no están distorsionados por prácticas discriminatorias. 
El segundo término captura el efecto rendimientos puesto que recoge la contribución de las diferencias en los mismos al gap salarial en el cuantil $\theta^{22}$.

El procedimiento completo descrito previamente se ha replicado 250 veces para obtener desviaciones típicas de tipo bootstrap en la medición de la contribución de dichos componentes.

Se aplica una descomposición similar a los residuos salariales obtenidos después de corregir por antigüedad en una QR. La idea subyacente detrás de este segundo gap contrafactual es la de chequear si el patrón de suelos resulta menos intenso cuando se omite el efecto de la antigüedad. Si la pendiente del gap a lo largo de la distribución se aplana, ello será señal de que la antigüedad es uno de los factores clave a la hora de explicar el fenómeno de los suelos. Por contra, si permanece de forma similar, la antigüedad no será una explicación relevante. Adicionalmente, con el fin de comparar el papel de la antigüedad con el de tener un contrato temporal/permanente, se efectúa un ejercicio similar, esta vez con los residuos salariales controlando únicamente por tipo de contrato en la QR.

\subsection{Resultados de la descomposición MM}

El Cuadro 4 presenta los resultados de la descomposición MM cuando se utilizan todas las variables explicativas, mientras que la Figura 3 ofrece el gap original y los tres gaps contrafactuales (la línea continua con todas las variables explicativas y las dos líneas discontinuas con los residuos salariales). Aunque implementar esta descomposición con las ecuaciones salariales corregidas de sesgo de selección solo añade una pequeña carga computacional adicional al procedimiento de MM (ya que la estimación del single-index solo tiene que hacerse una vez; véase Albrecht et al, 2005), hemos preferido reportar únicamente los resultados para las ecuaciones sin corrección. Procedemos así por dos razones. En primer lugar, porque el patrón de suelos persiste tras controlar por selección, como se discutió en la sección 3. En segundo lugar, porque puede argumentarse que la parte de corrección por selección muestral de la descomposición MM no es realmente necesaria ya que la distribución contrafactual estimada se construye a partir de los coeficientes de la QR de los hombres, y éstos es poco probable que estén sesgados por la presencia de selección.

Se observa que el gap contrafactual es claramente decreciente a lo largo de la distribución, alcanzando un mínimo de cerca del $50 \%$ en el cuantil $75^{\circ}$, en línea con el

22 Nótese que implementando esta descomposición, en contraste con la utilizada en Albrecht et al. (2003), estamos evaluando la diferencia de características con los rendimientos de los hombres. Si intercambiamos el papel de hombres y mujeres en la descomposición MM, que es lo que hacen estos autores, podríamos obtener una evaluación alternativa del diferencial de características usando los rendimientos femeninos,, de forma que el componente de Rendimientos sea evaluado con la muestra de hombres en cada cuantil. Los resultados de esta descomposición alternativa no se presentan al comprobarse que son cualitativamente parecidos a la hora de explicar la parte no explicada del gap salarial. 
fenómeno de los suelos. Esto es, mientras que el gap original se explica básicamente por las diferencias en rendimientos en la parte baja de la distribución, las diferencias en las características observables explican alrededor de la mitad del gap en la parte alta. Resulta interesante comprobar que el gap contrafactual de los residuos salariales tras corregir por antiguiedad es mucho más plano. Por el contrario, el gap contrafactual después de corregir por tipo de contrato se parece bastante al obtenido con todas las variables explicativas. Por tanto, de acuerdo con nuestra explicación, la discriminación estadística parece ser un factor determinante a la hora de generar un mayor gap en la parte baja de la distribución, un aspecto sobre el que se proporciona mas evidencia favorable en la siguiente sección utilizando un enfoque econométrico diferente.

\section{CUADRO 4}

GAPS CONTRAFACTUALES ENTRE GÉNEROS (GRUPO L. ESPAÑA 1999)

\begin{tabular}{|c|c|c|c|c|c|c|}
\hline & Media & $\theta=10$ & $\theta=25$ & $\theta=50$ & $\theta=75$ & $\theta=90$ \\
\hline Gap observado & 22,73 & 33,33 & 24,71 & 17,31 & 16,82 & 18,94 \\
\hline Gap contrafactual & $\begin{array}{l}17,08 \\
6(120)\end{array}$ & $\mathbf{3 1 , 3 4}$ & 21,54 & $\begin{array}{r}\mathbf{1 1 , 1 8} \\
(1,52)\end{array}$ & $\begin{array}{c}8,76 \\
156\end{array}$ & $\begin{array}{r}\mathbf{9 , 2 8} \\
(2,26)\end{array}$ \\
\hline$\%$ & 75,1 & 94 & 87,2 & 74,6 & 52,1 & 49 \\
\hline
\end{tabular}

Nota: Desviación estándar en paréntesis. Las desviaciones estándar se han obtenido mediante 250 replicaciones de la descomposición MM.

FIGURA 3

GAP SALARIAL (OBSERVADO CONTRAFACTUAL) GRUPO L. ESPAÑA - 1999

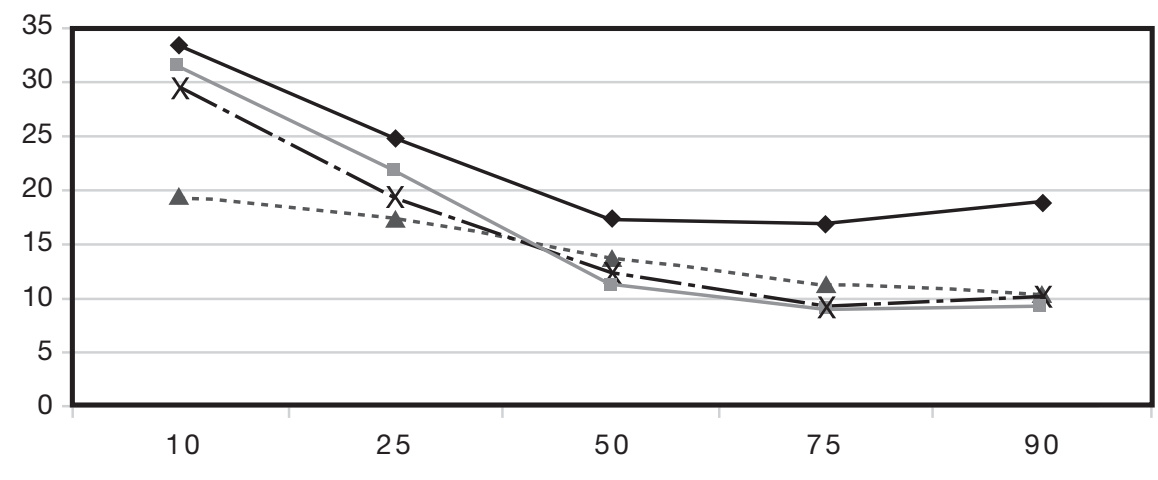

Cuantiles 


\section{Contraste de la hipótesis de discriminación estadística con datos de panel}

En las secciones anteriores hemos utilizado QR para argumentar en favor de la hipótesis de discriminación estadística a la hora de explicar el fenómeno de los suelos en el grupo L bajo el supuesto de que la edad/experiencia y la antigüedad aumentan a lo largo de la distribución salarial (véase Cuadro 2). Sin embargo esta conjetura podría reforzarse si fuéramos capaces de observar a los individuos en sus puestos de trabajo a lo largo del tiempo.

Afortunadamente ello es posible explotando la dimensión de panel del PHOGUE en sus ocho olas disponibles (1994-2001). La estimación con datos de panel (en concreto, estimación con efectos fijos) de ecuaciones salariales similares a las estimadas previamente - esta vez, en términos reales - puede usarse para contrastar la significación estadística y el signo de la interacción entre antigüedad y género (mujer=1) para cada grupo educativo, y para trabajadores jóvenes y adultos, respectivamente. Puesto que la estimación con efectos fijos remueve toda la heterogeneidad individual inobservable que no varíe en el tiempo, nos permitirá analizar de forma más directa el efecto de una mayor antigüedad sobre el rendimiento salarial en grupos de trabajadores con diferentes niveles educativos.

Puesto que nuestra explicación para el fenómeno de los suelos descansa en un comportamiento (estadísticamente) discriminatorio de los empresarios en contra de la mujeres de tipo L, debido a la percepción existente de una menor estabilidad en el empleo, deberíamos observar unos rendimientos iniciales de la antigüedad de las mujeres más elevados que los de los hombres en este grupo educativo, conforme desaparece dicha incertidumbre. Estas diferencias, sin embargo, no serían relevantes para los trabajadores en el grupo $\mathrm{H}$ donde tanto hombres como mujeres presentan altas tasas de participación laboral. Nuestra estrategia empírica se basa en la estimación por separado y conjuntamente de ecuaciones salariales para ambos géneros. En la estimación conjunta, todas las variables explicativas se interaccionan con una variable ficticia para las mujeres. De esta manera, el coeficiente de la interacción Antigüedad* Mujer para el grupo L debería ser positiva y estadísticamente significativa, mientras que no sería significativa para el grupo H. Además, el rendimiento de un año adicional de antigüedad para una mujer de tipo L debería aumentar bastantes más cuando sean jóvenes que cuando tengan una edad más avanzada ya que, en su juventud, es más probable que las mujeres se encuentren inmersas en el cuidado de sus hijos lo que aumenta su probabilidad de abandono. Por tanto, como un contraste adicional de nuestros resultados, también se lleva a cabo la estimación con efectos fijos dividiendo la muestra en dos grupos de edad: menores de 36 y mayores de 40 años en la primera ola del PHOGUE ${ }^{23}$. Se esperaría obtener un coeficiente del término de interacción descrito previamente mucho mayor para el primer que para el segundo grupo. Finalmente, para controlar por sesgos de selección en la muestra de trabajadores de

23 Pequeños cambios en los límites de edad para cada grupo no cambian significativamente los resultados. 
tipo L, se implementa el tradicional procedimiento bietápico de la corrección de Heckman donde, para estimar el probit inicial de participación separadamente para hombres y mujeres (no reportado), se usan variables de identificación similares a las discutidas en el Cuadro 3c.

El Cuadro 5a presenta los estadísticos descriptivos relativos a los salarios (en logs.), edad y antigüedad de la muestra al completo. Solamente se consideran aquellos trabajadores que son observados dos veces en el mismo empleo con el fin de estimar el efecto de cambios en la antigüedad sobre los salarios. La muestra final consiste en un panel desequilibrado con 21.657 observaciones, de las cuales 11.678 pertenecen al grupo L y 17. 535 al grupo de menores de 36 años de edad en 1994. En el grupo de mujeres jóvenes (primer panel del Cuadro 5a), se observa que el gap medio entre géneros es superior en el grupo L que en el grupo $\mathrm{H}$ ( 0.17 frente a 0.07$)$, los hombres tienen una edad superior en 0.5 años a las mujeres en ambos grupos, y la diferencia en antigüedad es mayor en el grupo L (en promedio, los hombre tienen una antigüedad cerca de 0.5 años superior a la de las mujeres) que en el grupo $\mathrm{H}$ (en promedio, los hombre tienen una antigüedad cerca de 0.33 años superior a la de las mujeres).

Los resultados de la estimación con efectos fijos de las ecuaciones salariales para los trabajadores jóvenes y más adultos se ofrecen en los Cuadros 5 b y $5 \mathrm{c}$, respectivamente. Para cada grupo de edad y para cada nivel educativo, se presentan estimaciones separadamente por género además de la estimación conjunta donde todas las variables explicativas se interaccionan con una variable ficticia para las mujeres. Se reportan los efectos de la edad (y su cuadrado) y de la antigüedad (y su cuadrado) de forma separada, así como de sus interacciones con la variable ficticia de mujeres en

\section{CUADRO 5a}

MEDIAS Y D.T. DE (LOG.) SALARIOS REALES, EDAD Y ANTIGÜEDAD (EN AÑOS)

\begin{tabular}{|l|c|c|c|c|c|c|c|c|}
\hline \multirow{4}{*}{} & \multicolumn{3}{|c|}{$\begin{array}{c}\text { Trabajadores menores de 36 años } \\
\text { en la primera ola (1994) }\end{array}$} & \multicolumn{3}{c|}{$\begin{array}{c}\text { Trabajadores mayores de 40 años } \\
\text { en la primera ola (1994) }\end{array}$} \\
\cline { 2 - 9 } & \multicolumn{2}{|c|}{ Hombres } & \multicolumn{2}{c|}{ Mujeres } & \multicolumn{2}{c|}{ Hombres } & \multicolumn{2}{c|}{ Mujeres } \\
\hline Educación & $\mathbf{L}$ & $\mathbf{H}$ & $\mathbf{L}$ & $\mathbf{H}$ & $\mathbf{L}$ & $\mathbf{H}$ & $\mathbf{L}$ & $\mathbf{H}$ \\
\hline \multirow{2}{*}{ Ln.) Salario } & 1,82 & 2,21 & 1,64 & 2,14 & 1,92 & 2,76 & 1,72 & 2,48 \\
& $(0,41)$ & $(0,49)$ & $(0,43)$ & $(0,49)$ & $(0,38)$ & $(0,56)$ & $(0,45)$ & $(0,42)$ \\
Edad & 28,77 & 30,62 & 28,18 & 30,16 & 50,45 & 49,21 & 49,84 & 47,63 \\
& $(5,88)$ & $(4,87)$ & $(5,62)$ & $(4,76)$ & $\begin{array}{c}(5,83) \\
(5,65)\end{array}$ & $\begin{array}{c}(5,77) \\
(4,50)\end{array}$ \\
Antigüedad & 3,51 & 4,42 & 3,03 & 4,08 & 4,65 & 7,78 & 5,36 & 8,19 \\
& $(4,33)$ & $(4,45)$ & $(3,86)$ & $(4,31)$ & $(4,88)$ & $(5,46)$ & $(4,91)$ & $(5,63)$ \\
$N^{o}$ obs. & 7.933 & 2.909 & 3.805 & 2.888 & 2.266 & 438 & 1.174 & 244 \\
\hline
\end{tabular}

Nota. L: Secundaria a menos es el máximo nivel educativo alcanzado. H: Terciaria es el máximo nivel educativo alcanzado. 
CUADRO 5b

REGRESIONES (LOG.) SALARIO REAL - TRABAJADORES MENORES DE 36 AÑOS EN LA PRIMERA OLA (1994). ESTIMACIONES POR EFECTOS FIJOS CON CORRECCIÓN DE SESGOS DE SELECCIÓN

\begin{tabular}{|c|c|c|c|c|c|c|c|c|}
\hline \multirow[b]{2}{*}{ Educación } & \multicolumn{3}{|c|}{ Hombres } & \multicolumn{3}{|c|}{ Mujeres } & \multicolumn{2}{|c|}{$\begin{array}{c}\text { H \& M } \\
\text { conjuntamente }\end{array}$} \\
\hline & Todos & $\mathbf{L}$ & $\mathbf{H}$ & Todos & $\mathbf{L}$ & $\mathbf{H}$ & $\mathbf{L}$ & $\mathbf{H}$ \\
\hline Edad & $\begin{array}{c}0,173 \\
(0,008)\end{array}$ & $\begin{array}{c}0,169 \\
(0,009)\end{array}$ & $\begin{array}{c}0,185 \\
(0,024)\end{array}$ & $\begin{array}{c}0,134 \\
(0,011)\end{array}$ & $\begin{array}{c}0,139 \\
(0,014)\end{array}$ & $\begin{array}{c}0,127 \\
(0,022)\end{array}$ & $\begin{array}{c}0,169 \\
(0,009)\end{array}$ & $\begin{array}{c}0,186 \\
(0,023)\end{array}$ \\
\hline Edad & $\begin{array}{c}-0,002 \\
(0,0001)\end{array}$ & $\begin{array}{c}-0,002 \\
(0,0001)\end{array}$ & $\begin{array}{c}-0,002 \\
(0,0004)\end{array}$ & $\begin{array}{c}-0,001 \\
(0,0002)\end{array}$ & $\begin{array}{c}-0,002 \\
(0,0002)\end{array}$ & $\begin{array}{c}-0,001 \\
(0,0004)\end{array}$ & $\begin{array}{c}-0,002 \\
(0,0001)\end{array}$ & $\begin{array}{c}-0,002 \\
(0,0003)\end{array}$ \\
\hline Antigüedad & $\begin{array}{c}0,009 \\
(0,002)\end{array}$ & $\begin{array}{c}0,004 \\
(0,003)\end{array}$ & $\begin{array}{c}0,019 \\
(0,006)\end{array}$ & $\begin{array}{c}0,017 \\
(0,003)\end{array}$ & $\begin{array}{c}0,018 \\
(0,005)\end{array}$ & $\begin{array}{c}0,016 \\
(0,006)\end{array}$ & $\begin{array}{c}0,004 \\
(0,003)\end{array}$ & $\begin{array}{c}0,019 \\
(0,005)\end{array}$ \\
\hline Antigüedad & $\begin{array}{l}-0,0006 \\
(0,0002)\end{array}$ & $\begin{array}{l}-0,0003 \\
(0,0002)\end{array}$ & $\begin{array}{c}-0,001 \\
(0,0002)\end{array}$ & $\begin{array}{c}-0,001 \\
(0,0002)\end{array}$ & $\begin{array}{c}-0,001 \\
(0,0003)\end{array}$ & $\begin{array}{c}-0,002 \\
(0,0004)\end{array}$ & $\begin{array}{c}-0,003 \\
(0,0002)\end{array}$ & $\begin{array}{c}-0,001 \\
(0,0004)\end{array}$ \\
\hline $\begin{array}{l}\text { Mujer* } \\
\text { Edad }\end{array}$ & - & - & - & - & - & - & $\begin{array}{l}-0,030 \\
(0,016)\end{array}$ & $\begin{array}{l}-0,058 \\
(0,033)\end{array}$ \\
\hline $\begin{array}{l}\text { Mujer* } \\
\text { Edad2 }\end{array}$ & - & - & - & - & - & - & $\begin{array}{c}0,0003 \\
(0,0002)\end{array}$ & $\begin{array}{c}0,0009 \\
(0,0005)\end{array}$ \\
\hline $\begin{array}{l}\text { Mujer* } \\
\text { Antig. }\end{array}$ & - & - & - & - & - & - & $\begin{array}{c}0,018 \\
(0,006)\end{array}$ & $\begin{array}{l}-0,003 \\
(0,008)\end{array}$ \\
\hline $\begin{array}{l}\text { Mujer* } \\
\text { Antig2 }\end{array}$ & - & - & - & - & - & - & $\begin{array}{c}-0,001 \\
(0,0004)\end{array}$ & $\begin{array}{l}-0,0007 \\
(0,0006)\end{array}$ \\
\hline $\begin{array}{l}\text { Término de } \\
\text { selección }\end{array}$ & $\begin{array}{l}-0,016 \\
(0,036)\end{array}$ & $\begin{array}{l}-0,077 \\
(0,051)\end{array}$ & $\begin{array}{c}0,032 \\
(0,094)\end{array}$ & $\begin{array}{c}0,164 \\
(0,050)\end{array}$ & $\begin{array}{c}0,439 \\
(0,197)\end{array}$ & $\begin{array}{l}-0,605 \\
(0,558)\end{array}$ & $\begin{array}{l}-0,077 \\
(0,050)\end{array}$ & $\begin{array}{c}0,032 \\
(0,091)\end{array}$ \\
\hline $\begin{array}{l}\text { Término de } \\
\text { Sel* Mujer }\end{array}$ & - & - & - & - & - & - & $\begin{array}{c}0,516 \\
(0,312)\end{array}$ & $\begin{array}{l}-0,637 \\
(0,583)\end{array}$ \\
\hline No. obs & 10.842 & 7.933 & 2.909 & 6.693 & 3.805 & 2.888 & 11.738 & 5.797 \\
\hline No. grupos & 2.513 & 2.005 & 835 & 1.689 & 1.141 & 810 & 3.146 & 1.645 \\
\hline
\end{tabular}

Nota: Todas las regresiones incluyen 6 variables ficticias de región, 2 de industria y otra de grado de supervisión. Se omiten los controles por ocupación ya que en casi todos los casos no exhiben variaciones a lo largo del tiempo para cada individuo. En la estimación conjunta de hombres y mujeres, exhibida en las dos últimas columnas, todas las variables explicativas se interaccionan con la variable artificial Mujer.

la estimación conjunta ${ }^{24}$. Tal como se muestra en el Cuadro $5 b$, los rendimientos de la edad son menores para las mujeres en ambos grupos educativos. Por lo que respecta a la antigüedad, se observa - tanto en las ecuaciones estimadas por separado como conjuntamente - que los rendimientos de las mujeres de tipo L son significativamente más altas que las de los hombres. Los coeficientes estimados del término Antigüe-

24 La edad reemplaza a la experiencia potencial como control ya que la edad a la que un individuo comienza su primer empleo no cambia con el tiempo. 


\section{CUADRO 5c}

REGRESIONES (LOG.) SALARIO REAL - TRABAJADORES MAYORES DE 40 AÑOS EN LA PRIMERA OLA (1994). ESTIMACIONES POR EFECTOS FIJOS CON CORRECCIÓN DE SESGOS DE SELECCIÓN

\begin{tabular}{|c|c|c|c|c|c|c|c|c|}
\hline \multirow[b]{2}{*}{ Educación } & \multicolumn{3}{|c|}{ Hombres } & \multicolumn{3}{|c|}{ Mujeres } & \multicolumn{2}{|c|}{$\begin{array}{c}\text { H \& M } \\
\text { conjuntamente }\end{array}$} \\
\hline & Todos & $\mathbf{L}$ & $\mathbf{H}$ & Todos & $\mathbf{L}$ & $\mathbf{H}$ & $\mathbf{L}$ & $\mathbf{H}$ \\
\hline \multirow[t]{2}{*}{ Edad } & 0,016 & 0,005 & 0,065 & 0,035 & 0,031 & 0,040 & 0,005 & 0,063 \\
\hline & $(0,021)$ & $(0,024)$ & $(0,057)$ & $(0,031)$ & $(0,036)$ & $(0,097)$ & $(0,024)$ & $(0,053)$ \\
\hline \multirow[t]{2}{*}{ Edad } & 0,00008 & 0,0002 & $-0,0004$ & $-0,0002$ & $-0,0002$ & 0,0009 & 0,0001 & $-0,0003$ \\
\hline & $(0,0002)$ & $(0,0002)$ & $(0,0005)$ & $(0,0003)$ & $(0,0003)$ & $(0,0008)$ & $(0,0002)$ & $(0,0005)$ \\
\hline \multirow[t]{2}{*}{ Antigüedad } & 0,013 & 0,010 & 0,019 & $-0,006$ & 0,0007 & $-0,038$ & 0,010 & 0,019 \\
\hline & $(0,004)$ & $(0,005)$ & $(0,013)$ & $(0,006)$ & $(0,007)$ & $(0,0006)$ & $(0,004)$ & $(0,012)$ \\
\hline \multirow[t]{2}{*}{ Antigüedad } & $-0,0005$ & $-0,0002$ & $-0,002$ & 0,0003 & 0,0001 & 0,001 & $-0,0002$ & $-0,001$ \\
\hline & $(0,0002)$ & $(0,0003)$ & $(0,0006)$ & $(0,0003)$ & $(0,0004)$ & $(0,0006)$ & $(0,0003)$ & $(0,0006)$ \\
\hline Mujer* & & & & & & & 0,026 & $-0,025$ \\
\hline Edad & & & & & & & $(0,044)$ & $(0,123)$ \\
\hline Mujer* & & & & & & & $-0,0004$ & 0,0004 \\
\hline Edad2 & & & & & & & $(0,0004)$ & $(0,001)$ \\
\hline Mujer* & - & - & - & - & - & - & $-0,009$ & $-0,057$ \\
\hline Antig. & & & & & & & $(0,009)$ & $(0,021)$ \\
\hline Mujer* & - & - & - & - & - & - & 0,0003 & 0,003 \\
\hline Antig2 & & & & & & & $(0,0005)$ & $(0,0009)$ \\
\hline Término de & 0,018 & $-0,026$ & $-0,404$ & $-0,055$ & $-0,119$ & 2,28 & $-0,026$ & 0,403 \\
\hline selección & $(0,110)$ & $(0,164)$ & $(0,455)$ & $(0,225)$ & $(0,97)$ & $(4,00)$ & $(0,163)$ & $(0,429)$ \\
\hline Término de & - & - & - & - & - & - & $-0,092$ & 2,69 \\
\hline Sel* Mujer & & & & & & & $(0,995)$ & $(4,55)$ \\
\hline No. obs & 2.704 & 2.266 & 438 & 1.414 & 1.174 & 240 & 3.440 & 678 \\
\hline No. grupos & 592 & 521 & 104 & 328 & 300 & 51 & 821 & 155 \\
\hline
\end{tabular}

Nota: Todas las regresiones incluyen 6 variables ficticias de región, 14 de ocupación, 2 de industria y otra de grado de supervisión. Se excluyen los trabajadores con antigüedad superior a 15 años ya que, para ellos, la variable antigüedad está truncada en 15 años. En la estimación conjunta de hombre sy mujeres, exhibida en las dos últimas columnas, todas las variables explicativas se interaccionan con la variable artificial Mujer.

$d a d^{*}$ Mujer y su cuadrado resultan ser altamente significativas y el efecto diferencial es cóncavo. Por contra, los correspondientes efectos estimados para el grupo $\mathrm{H}$ no son significativos. Por consiguiente, ambos resultados confirman fehacientemente el papel de la discriminación estadística en la evolución del rendimiento de la antigüedad a la hora de explicar el fenómeno de los suelos. Ello se confirma todavía más al observar los resultados en el Cuadro 5c para los trabajadores de edad más avanzada, donde el efecto diferencial por género en los correspondientes rendimientos para ambos grupos educativos es no significativo. 
Para evaluar el efecto diferencial de la antigüedad entre las mujeres y los hombres jóvenes del grupo L, se pueden utilizar los coeficientes estimados del término de interacción Antigüedad*Mujer y su cuadrado en la penúltima columna del Cuadro 5b. Con una antigüedad media de 3.2 años (véase el Cuadro 5a), la diferencia de rendimientos es de 3.46 puntos porcentuales al año, lo que supone un gap sustancial. Todavía mas importante es que el efecto acumulativo de las diferencias de los rendimientos de la antigüedad y su cuadrado se maximiza a los 9 años, antigüedad para la que dicha diferencia alcanza 8.1 puntos porcentuales.

Finalmente, otro resultado destacable es que la probabilidad de participar ejerce un efecto positivo sobre los salarios únicamente para las mujeres jóvenes del grupo L, lo que significa que estas mujeres (observadas al menos dos veces en su empleo) no son una muestra aleatoria de la población de todas las mujeres del grupo L, ya que aquellas tienen salarios superiores. En consecuencia, como se indicó en la discusión de los resultados de las QR, ello implica que el patrón de suelos sería todavía más intenso si nuestra muestra de mujeres de tipo L fuera una muestra aleatoria de dicha población. Nótese, por último, que en la medida en que la heterogeneidad inobservable se supone invariante en el tiempo, la estimación por efectos fijos debilita otras explicaciones alternativas de los suelos basadas en que la heterogeneidad afecte a las mujeres (relativamente a los hombres) de forma desproporcionada.

\section{Conclusiones e implicaciones de política laboral}

En este trabajo, se ha analizado la evolución de los gaps salariales entre géneros a lo largo de la distribución salarial en España, utilizando técnicas econométricas de QR y datos de panel. Nuestro principal resultado es que detrás de la evolución irregular del gap para la muestra completa de individuos, se encuentra una diferencia apreciable entre los patrones de los gaps salariales cuando se distingue entre grupos de trabajadores con bajo $(\mathrm{L})$ y alto $(\mathrm{H})$ nivel educativo. Mientras que para el grupo $\mathrm{H}$, el gap decrece a lo largo de la distribución (techos de cristal), como ocurre en muchos otros países, se vuelve fuertemente decreciente en el grupo L (patrón de sue$l o s)$. En concreto, esta última evolución - no documentada anteriormente - contrasta con la existente en los países del centro y norte de la UE, donde el gap crece a lo largo de la distribución, independientemente del grupo educativo que se examine. En este sentido, se presenta evidencia que apunta a un patrón similar de suelos en la mayoría de los países del sur de la UE, como Grecia o Italia, donde la participación laboral de las mujeres del grupo L todavía es reducida.

Se argumenta que la presencia de selección muestral no aleatoria no puede explicar el nuevo fenómeno de los suelos. Nuestra explicación alternativa se basa en la existencia de discriminación estadística cuando los costes de formación se financian entre empresarios y trabajadores. Debido a la baja tasa de actividad de las mujeres del grupo L, los empresarios pueden usar la discriminación estadística para reducir los salarios de las mujeres frente a los de los hombres, percibidos como más estables en el 
empleo, en la parte baja de la distribución ya que esperan una mayor tasa de abandono femenina que comprometa la parte de financiación empresarial de la formación específica. Sin embargo, conforme aumenta la antigüedad de las mujeres en el puesto de trabajo, se convierten en más «fiables» a los ojos de los empresarios, de manera que sus salarios convergen con los de los hombres, a igualdad de características productivas observables. Esta hipótesis de discriminación estadística se ve reforzada cuando explotamos la dimensión de panel del PHOGUE, siguiendo a mujeres en sus mismos empleos y observando cómo evoluciona el gap entre géneros en esa situación. Encontramos evidencia concluyente de que el rendimiento en el mercado laboral a una mayor antigüedad en el puesto de trabajo es mayor para las mujeres de tipo L que para los hombres en dicho grupo, implicando menores gaps cuando la antigüedad de ambos aumenta. Este resultado, sin embargo, no se cumple para las mujeres en el grupo $\mathrm{H}$, menos propensas al abandono tras haber invertido fuertemente en su educación, ni tampoco para las mujeres mayores de 40 años en el grupo L, menos propensas a abandonar por razones derivadas del nacimiento y cría de sus hijos.

Las implicaciones de la presencia de suelos para las políticas laborales y sociales pueden ser muy interesantes. Por ejemplo, podrían conducir a la existencia de un modelo de equilibrios múltiples de gaps entre géneros en términos salariales y de tasas de participación laboral. Si las creencias culturales o un gasto social reducido en ayudas a las familias conllevan una menor tasa de actividad femenina en el grupo L, nuestros argumentos predicen que esta situación inicial aumentará el gap salarial a favor de los hombres, lo que, a su vez, agravará la baja participación femenina Por tanto, ambos efectos conducen a un equilibrio «malo» con altas diferencias en tasas de actividad y salarios. Por el contrario, si no existe discriminación social y/o la ayuda familiar es generosa, la alta participación femenina conllevará menores gaps salariales, lo que realimentará una mayor tasa de actividad femenina, conduciendo a la economía a un «buen» equilibrio. Esta última situación parece corresponder a lo que acontece en los países nórdicos, mientras que la primera caracteriza lo que ocurre en los países antes mencionados del sur de la UE. En estas circunstancias, la políticas públicas en favor de la reconciliación de la vida familiar y laboral podrían trasladar a la economía desde un equilibrio «malo» a uno «bueno» no sólo, como se piensa convencionalmente, mediante un aumento de la participación laboral femenina sino, como se enfatiza en este trabajo, a través de la reducción del gap salarial entre hombres y mujeres. Un análisis de estas implicaciones para el diseño de políticas sociales cuando la participación se determina en forma endógena es parte de nuestra agenda de investigación actual.

\section{Apéndice}

\section{A.1: Definición de variables}

Las variables en las QR proceden de la (sexta) ola del PHOGUE correspondiente a 1999. Nuestra muestra está compuesta por asalariados a tiempo completo (con al me- 
nos 1.560 horas de trabajo al año o 30 horas por semana en promedio). A continuación se ofrece una descripción mas detallada de las variables usadas en las estimaciones.

Salario bruto por hora: El PHOGUE contiene información sobre la renta laboral media (bruta y neta) de los asalariados. La renta laboral incluye pagas extra (divididas por el número de meses de trabajo) y remuneración por horas extraordinarias. Cuando un trabajador tiene más de un empleo, solo se considera el empleo principal. Se conocen las horas semanales de trabajo y las horas extraordinarias. Se ha establecido una cota superior de 60 horas para esta variable con el fin de minimizar sesgos en las horas declaradas por el trabajador. Esta corrección afecta al 2\% de los hombres y al $0.9 \%$ de las mujeres en la muestra. Por tanto, el salario bruto por hora se obtiene dividiendo el salario bruto mensual por 52/12 y multiplicando esta ratio por el número de horas trabajadas semanalmente en el empleo principal.

Experiencia: Se define como la edad actual menos la edad a la que el individuo comenzó a trabajar.

Exp*Hijos: Se define como la interacción entre experiencia y una variable binaria que toma el valor 1 cuando un individuo tiene hijos dependientes (de 0 a 16 años). En la ecuación de participación del Cuadro 3a, se consideran separadamente los casos en que los hijos tienen entre 0 y 11 años (Exp*Hijos 0-11) y entre 12 y 16 años (Exp*Hijos 12-16), pero únicamente la primera interacción resultó ser significativa.

Educación secundaria: Se define como una variable ficticia de haber completado la educación secundaria.

Características individuales: Variables ficticias de estado civil, condición de inmigrante, lugar de residencia y tamaño del municipio.

Tipo de Contrato: Temporal o permanente.

Sector: Privado o público.

Tareas de supervisión: Directivo o encargado, supervisor de al menos otro empleado sin responsabilidad sobre el resto de empleados.

Antiguiedad: Se obtiene como la diferencia entre el año de la muestra y el año en que comenzó en el puesto de trabajo actual.

Tamaño de la empresa: De 1 a 4 empleados, de 5 a 19 empleados, de 20 a 49 empleados, de 50 a 99 empleados, de 100 a 499 empleados y más de 500 empleados.

Ocupación: Se han considerado quince grupos ocupacionales correspondientes a un nivel intermedio de desagregación de la clasificación ISCO-88 (COM). La lista es: Legisladores, directores y gerentes ( $\mathrm{OC} 1)$; Profesionales de nivel superior; (OC2); Profesionales de la enseñanza (OC3); Otros profesionales (OC4); Profesionales de nivel medio (OC5); Profesionales de nivel medio en la enseñanza (OC6); Administrativos (OC7), Dependientes de comercios y asimilados (OC8); Trabajadores de los servicios de protección y seguridad (OC9); Trabajadores cualificados de la agricultura y pesca (OC10); Trabajadores cualificados de la construcción, industrias extractivas y metalurgia (OC11); Trabajadores cualificados del metal, maquinaria móvil y artes gráficas (OC12); Operadores de instalaciones industriales (OC13); Trabajadores no cualificados en servicios (OC14); y Peones en la agricultura, pesca, construcción, industria manufacturera y transportes (OC15). 


\section{CUADRO A1}

TASAS DE ACTIVIDAD POR NIVEL EDUCATIVO (2002)

\begin{tabular}{|l|c|c|c|c|c|c|}
\hline Países & \multicolumn{3}{|c|}{ Hombres } & \multicolumn{3}{c|}{ Mujeres } \\
\hline & $\begin{array}{c}\text { Inferior a } \\
\text { secundaria }\end{array}$ & Secundaria & Terciaria & $\begin{array}{c}\text { Inferior a } \\
\text { Secundaria }\end{array}$ & Secundaria & Terciaria \\
\hline Dinamarca & 75.4 & 87.3 & 92.7 & 55.8 & 80.7 & 88.4 \\
Suecia & 78.0 & 87.9 & 90.4 & 65.1 & 83.4 & 88.1 \\
R.U. & 65.9 & 88.1 & 92.2 & 50.7 & 76.4 & 87.3 \\
Grecia & 81.0 & 88.5 & 89.7 & 42.1 & 57.2 & 82.4 \\
Italia & 75.5 & 86.1 & 90.9 & 34.8 & 67.9 & 82.7 \\
España & 83.5 & 90.1 & 91.9 & 42.3 & 67.6 & 83.1 \\
\hline
\end{tabular}

Fuente: OECD, Employment Outlook, 2002.

\section{Referencias bibliográficas}

[1] ABADIE, A. (1997): "Changes in the Spanish Labor Income Structure during the 1980`s: A Quantile Regression Approach», Investigaciones Económicas, 21, 253-272.

[2] ALBRECHT, J., A. BJÖRKLUND y S. VROMAN (2003): «Is There a Glass Ceiling in Sweden?», Journal of Labor Economics, 21, 145-177.

[3] ALBRECHT, J., A. VAN VUUREN, y S. VROMAN (2004): «Decomposing the Gender Gap in the Netherlands with Sample Selection Adjustments», (mimeo).

[4] ACEMOGLU, D. y J.S. PISCHKE (1998): «Why Do Firms Train: Theory and Evidence», Quarterly Journal of Economics, 113, 79-111.

[5] ARELLANO, M. y O. BOVER (1995): «Female Labour Force Participation in the 1980s: The Case of Spain», Investigaciones Económicas, 29, 171- 194.

[6] BLAU, .F. y L. KAHN (2000): «Gender Differences in Pay», Journal of Economic Perspectives, 14, 75-99

[7] BOOTH, A., M. FRANCESCONI y J. FRANK (2003): «A Sticky Floors Model of Promotion, Pay and Gender», European Economic Review, 47, 295- 322.

[8] BUCHINSKY, M. (1994): «Changes in the U.S. Wage Structure 1963-1987: An Application of Quantile Regression», Econometrica, 62, 405-458.

[9] BUCHINSKY, M. (1998a): «Recent Advances in Quantile Regression Models: A Practical Guideline for Empirical Research», The Journal of Human Resources, 33, 88126.

[10] BUCHINSKY, M. (1998b): «The Dynamics of Changes in the female Wage Distribution in the USA: A Quantile Regression Approach», Journal of Applied Econometrics, 13, 1-30.

[11] CHAMBERLAIN, G. (1994): «Quantile Regression, Censoring and the Structure of Wages», Advances in Econometrics, Sixth World Congress (Volume 1); C. A. Sims (ed.), Cambridge University Press. 
[12] DE LA RICA, S., DOLADO, J. y V. LLORENS (2005): « Ceilings and Floors: Gender Wage Gaps by Education in Spain», IZA DP no. 1483.

[13] DOLADO, J.J., GARCIA-SERRANO, C. y J.F. JIMENO (2002): « Drawing Lessons from the Boom of Temporary Jobs in Spain», The Economic Journal, 112, 270295.

[14] DOLADO, J.J., F. FELGUEROSO y J.F. JIMENO (2004): «Where do Women Work?: Analyzing Patterns in Occupational Segregation By Gender», Annales d'Economie et de Statistique, 71-72, 293-315.

[15] DOLADO, J.J. y V. LLORENS (2004): «Gender Gaps by Education in Spain: Glass Floors vs. Glass Ceilings», CEPR DP. 4203.

[16] FARBER, H. y R. GIBBONS (1996). «Learning and Wage Dynamics.» Quarterly Journal of Economics, 111, 1007-1047.

[17] FITZBERGER, B., KOENKER, R., y J.A.F. MACHADO (Eds.) (2001): Economic Applications of Quantile Regression, Physica-Verlag.

[18] GARCÍA, J., P.J. HERNÁNDEZ y A. LÓPEZ-NICOLÁS (2001): «How Wide is the Gap? An Investigation of Gender Wage Differences using Quantile Regression», Empirical Economics, 26, 149-168.

[19] GARDEAZÁBAL, J. y A. UGIDOS (2002): «A Measure of Gender Wage Discrimination at Quantiles», Journal of Population Economics, forthcoming.

[20] INSTITUTO DE LA MUJER (2005): Estudio sobre la Conciliación de la Vida Familiar y la Vida laboral en España: Situación Actual, Necesidades y Demanda, MTAS. (http://www.mtas.es/mujer/mujeres/estud_inves/estud_anterior04.htm.)

[21] KOENKER, R. y G. BASSETT (1978): «Regression Quantiles», Econometrica, 46, 33-50.

[22] KOENKER, R. y G. BASSETT (1982): «Robust Tests for Heteroskedasticity on Regression Quantiles», Econometrica, 50, 43-61.

[23] LAZEAR, E. y S. ROSEN (1990): «Male-Female Wage Differentials in Job Ladders», Journal of Labour Economics, 8, 106-123.

[24] MACHADO, J.A.F. y J. MATA (2005): «Counterfactual Decomposition of Changes in Wage Distributions using Quantile Regression», Journal of Applied Econometrics, 20, 445-465.

[25] MANNING, A. (2003): Monopsony in Motion, Princeton University Press.

[26] MINCER, J. y S. POLACHECK (1974): «Family Investment in Human Capital: Earnings of Women», Journal of Political Economy, 82, 76-108.

[27] NEWELL, A. y B. REILLY (2001): «The Gender Pay Gap in the Transition from Communism: Some Empirical Evidence», Economic Systems, 25, 287-304.

[28] OECD (2002): «Women at Work: Who Are They and How Are They Faring?», Employment Outlook, 63-125.

[29] OLIVETTI, C. y B. PETRONGOLO (2005): «Unequal Pay or Unequal Employment ?: A Cross-country analysis of Gender Gaps». (mimeo)

[30] POLACHECK, S. (1981): «Occupational Self-Selection: A Human Capital Approach to Sex Differences in Occupational Structure», Review of Economics and Statistics, 62, 60-69. 
